\title{
Penalization for birth and death processes
}

\author{
Pierre Debs and Mihai Gradinaru \\ Institut Élie Cartan Nancy, B.P. 239, \\ 54506 Vandouvre-lès-Nancy Cedex, France \\ \{Pierre.Debs, Mihai.Gradinaru\}@iecn.u-nancy.fr
}

\begin{abstract}
In this paper we study a transient birth and death Markov process penalized by its sojourn time in 0 . Under the new probability measure the original process behaves as a recurrent birth and death Markov process. We also show, in a particular case, that an initially recurrent birth and death process, behaves as an transient birth and death process after penalization with the event that it can reach zero in infinite time. We illustrate some of our results with the Bessel random walk example.
\end{abstract}

Key words: Birth and death Markov processes; penalization; sojourn time; Dynkin's formula; random walk; Brownian motion with drift; Bessel chain and process; change of probability.

2000 Mathematics Subject Classification: 60J80, 60J10, 60J27, 60J75, 60B10, 60G42, 60G44.

\section{Introduction}

Consider a transient Markov chain with positive states. Is it possible to force the chain to have an infinite number of visits at the state 0 ? Does one have a reasonable manner to do this? Conditionally to this behaviour, is the resulting chain recurrent or transient? These are some natural questions when studying penalization for Markov chains, that is to modify their laws in order to alter some of their properties. In the present paper by penalization one understands to condition by an event with probability zero, in our case, the event that the transient Markov chain returns infinitely often at 0 .

The penalization of a probability measure (for instance, the distribution of some Markov process) by some appropriate weights is the main tool of some classical probabilistic ideas as Doob's $h$-transform, Feynman-Kac transform or Girsanov transform. For instance, in the latter case, an exponential martingale occurs as weight factor and, under some natural conditions, a new probability measure is well defined. Since, dividing a probability distribution by a family of weights does not create necessarily a probability family of distributions, one needs to made some limiting procedure (often as the parameter goes to infinity). The main features of the penalization procedure have been pointed out in a series of papers by Roynette,Vallois,Yor (see [12] for a survey). Essentially, one considers continuous parameter Markov processes (Brownian motion or Bessel processes) and one penalizes by some function of local time, maximum, one-sided maximum etc.

For instance, one considers $\left(\Omega,\left\{\mathcal{F}_{t}\right\}_{0 \leq t \leq \infty},\left\{R_{t}\right\}_{t \geq 0}, \mathbb{P}\right)$ the canonical Bessel process of index $\alpha \in(-1,0)$, and one denotes $\left\{L_{t}\right\}_{t \geq 0}$ its local time at level 0 . Then one can be interested on the limit

$$
\lim _{t \rightarrow \infty} \frac{\mathbb{E}_{0}\left[\mathbb{1}_{A_{s}} h\left(L_{t}\right)\right]}{\mathbb{E}_{0}\left[h\left(L_{t}\right)\right]}=Q_{0}^{(h)}\left(A_{s}\right)=\mathbb{E}_{0}\left[\mathbb{1}_{A_{s}} M_{s}^{(h)}\right], A_{s} \in \mathcal{F}_{s} \quad(s \text { fixed }), h: \mathbb{R}_{+} \rightarrow \mathbb{R}_{+},
$$


and one proves that the process $\left\{M_{s}^{(h)}\right\}_{s \geq 0}$ is a martingale. Then one studies the canonical process $\left\{R_{t}\right\}_{t \geq 0}$ under the new probability $Q_{0}^{(h)}$ and some interesting properties of this process are pointed out.

The discrete parameter counterpart of the same problems was performed very recently by Debs [1] for random walks. For instance, if $\left(\Omega,\left\{\mathcal{F}_{n}\right\}_{0 \leq n \leq \infty},\left\{S_{n}\right\}_{n \geq 0}, \mathbb{P}\right)$ is the canonical standard random walk and if one calls the local time at 0 the following sequence

$$
\ell_{0}=0, \quad \ell_{n+1}=\ell_{n}+\mathbb{1}_{\left\{S_{n}=0\right\}}, n \geq 0,
$$

then one can be interested on the limit

$$
\begin{aligned}
& \lim _{p \rightarrow \infty} \frac{\mathbb{E}\left[\mathbb{1}_{A_{n}}\left(\left(h\left(\ell_{p}\right) \mathbb{1}_{\left\{S_{p}>0\right\}}+g\left(\ell_{p}\right) \mathbb{1}_{\left\{S_{p}<0\right\}}\right)\right)\right]}{\mathbb{E}\left[h\left(\ell_{p}\right) \mathbb{1}_{\left\{S_{p}>0\right\}}+g\left(\ell_{p}\right) \mathbb{1}_{\left\{S_{p}<0\right\}}\right]} \\
&=Q^{(h, g)}\left(A_{n}\right)=E\left[\mathbb{1}_{A_{n}} M_{n}^{(h, g)}\right], \quad A_{n} \in \mathcal{F}_{n} \quad(n \text { fixed }), g, h: \mathbb{N} \rightarrow \mathbb{R}_{+} .
\end{aligned}
$$

Again, one proves that $\left\{M_{n}^{(h, g)}\right\}_{n \geq 0}$ is a martingale and one studies the canonical sequence $\left\{S_{n}\right\}_{n \geq 0}$ under the probability $Q^{(\bar{h}, g)}$. Let us notice that in almost of cases considered in the cited works, one uses as important tools precise informations on the processes : explicit laws of hitting times, explicit martingales related to considered processes and so on. This allows to perform exact computations when proving certain properties.

In the present paper we consider the birth and death Markov chains. Recall that these processes have the property that only transitions to neighbouring states are possible. This model comes from applications in biology, the developing in time of a population of particles: each particle lives for a random length of time at the end of which it splits into two particles or dies. The process is quite simple and constitutes a generalisation of the standard random walk on $\mathbb{N}$ (in the discrete parameter setting) and have some similarities with Bessel processes (in the continuous parameter setting).

We address the previous simple questions, for birth and death Markov chains with discrete and continuous parameter. Precisely, we penalize the distribution of the transient birth and death process by the number of visits at the state 0 (which is like local time type penalization). When we force the chain to visit an infinitely often the state zero it is reasonable to think that what we get is a recurrent chain. Indeed, we prove that, under the new probability measure induced by penalization, the chain behaves as a recurrent birth and death chain. The same procedure works in continuous parameter setting. We point out that in [11] one studies the Bessel process of dimension greater than 2 (which is a transient process) penalized in some sense by its local time at 0 (more precisely, by its sojourn time in an interval, see also Remark 4.1 below).

Our approach is elementary in the discrete parameter setting, and it is based on Dynkin's formula for pure jump processes in the continuous parameter setting. We would like to stress that for both situations all we need is the transient feature of the process, but we do not need neither explicit expressions of transition probabilities (or rates), nor other explicit informations related to our processes as in previous cited works. In some sense this is one of the originalities of the present paper, besides the study of the penalization for birth and death chains.

Is it possible to penalize a recurrent birth and death chain in order to obtain a transient one? We give a particular example where we penalize a recurrent random walk by the event 
to reach zero in infinite time and we get a recurrent birth and death chain. Our proof for this case uses the expression of the law of the hitting time of zero for a biased random walk and we obtain a particular birth and death chain, the so-called Bessel random walk. For this reason it seems that the case of a general birth and death chain is more complicate. We illustrate some other phenomena by further study of Bessel random walks.

The paper is organised as follows : the main results concerning the penalization of transient birth and death chains, with discrete and also with continuous parameter, are stated in the next section. Section 2 also contains an example of penalization of recurrent birth and death chain. All the proofs are given in Section 3. The example of Bessel random walks, but also some other examples are discussed in the last section of the paper.

\section{Notations and main results}

\subsection{Discrete parameter setting}

Let $\left\{X_{n}\right\}_{n \geq 0}$ be a birth and death chain on the canonical probability space $\left(\Omega, \mathcal{F}_{\infty}, \mathbb{P}\right)$. In other words, $\left\{X_{n}\right\}_{n \geq 0}$ is a Markov chain, with state space $\mathbb{N}$ and having the transition probabilities given by:

$$
\begin{aligned}
p_{\ell}:=\mathbb{P}\left(X_{n+1}=\ell+1 \mid X_{n}=\ell\right), q_{\ell} & :=\mathbb{P}\left(X_{n+1}=\ell-1 \mid X_{n}=\ell\right), \\
r_{\ell} & :=\mathbb{P}\left(X_{n+1}=\ell \mid X_{n}=\ell\right)=1-p_{\ell}-q_{\ell}, \ell \geq 1,
\end{aligned}
$$

and

$$
p_{0}:=\mathbb{P}\left(X_{n+1}=1 \mid X_{n}=0\right), q_{0}:=0, r_{0}:=1-p_{0} .
$$

We will denote

$$
\gamma_{0}:=1, \gamma_{\ell}:=\prod_{k=1}^{\ell} \frac{q_{k}}{p_{k}}, \ell \geq 1,
$$

and it can proved (see for instance [6], p. 77) the following classical:

Proposition 2.1 The birth and death chain $\left\{X_{n}\right\}_{n \geq 0}$ is transient if and only if

$$
\sum_{\ell \geq 0} \gamma_{\ell}<\infty
$$

and in this case we will denote

$$
S:=\sum_{\ell \geq 0} \gamma_{\ell}
$$

Set

$$
U_{n}:=\sum_{k=0}^{n} \mathbb{1}_{\left\{X_{k}=0\right\}}, \quad 0 \leq n \leq \infty .
$$

We are able to state the first main result:

Theorem 2.1 Let $\left\{\mathcal{F}_{n}\right\}_{n \geq 0}$ be the natural filtration associated to the transient birth and death chain $\left\{X_{n}\right\}_{n \geq 0}$. 
1. For any integer $n \geq 0$ and any event $A_{n} \in \mathcal{F}_{n}$,

$$
\lim _{\kappa \rightarrow \infty} \frac{\mathbb{E}_{0}\left[\mathbb{1}_{A_{n}} \mathbb{1}_{\left\{U_{\infty} \geq \kappa\right\}}\right]}{\mathbb{E}_{0}\left[\mathbb{1}_{\left\{U_{\infty} \geq \kappa\right\}}\right]}=\mathbb{E}_{0}\left[\mathbb{1}_{A_{n}} M_{n}\right]
$$

where

$$
M_{n}:=\left(\frac{1}{S}\right)\left(1-\frac{p_{0}}{S}\right)^{-U_{n}+\mathbb{1}_{\left\{X_{n}=0\right\}}}\left(\sum_{\ell \geq X_{n}} \gamma_{\ell}\right) .
$$

2. $\left\{M_{n}\right\}_{n \geq 0}$ is a positive $\left\{\mathcal{F}_{n}\right\}$-martingale which tends toward zero, almost surely. Henceforth $\left\{M_{n}\right\}_{n \geq 0}$ is not uniformly integrable.

3. The equality $(2.7)$ induces a probability measure $Q_{0}$ on $\left(\Omega, \mathcal{F}_{\infty}\right)$ : for any integer $n \geq 0$ and any event $A_{n} \in \mathcal{F}_{n}$,

$$
Q_{0}\left(A_{n}\right):=\mathbb{E}_{0}\left[\mathbb{1}_{A_{n}} M_{n}\right] .
$$

Then, under $Q_{0},\left\{X_{n}\right\}_{n \geq 0}$ is a recurrent birth and death chain with transition probabilities given by:

$$
\begin{aligned}
& \tilde{p}_{\ell}=Q_{0}\left(X_{n+1}=\ell+1 \mid X_{n}=\ell\right):= p_{\ell}\left(\sum_{k \geq \ell+1} \gamma_{k}\right) /\left(\sum_{k \geq \ell} \gamma_{k}\right) \\
& \tilde{q}_{\ell}=Q_{0}\left(X_{n+1}=\ell-1 \mid X_{n}=\ell\right):=q_{\ell}\left(\sum_{k \geq \ell-1} \gamma_{k}\right) /\left(\sum_{k \geq \ell} \gamma_{k}\right), \\
& \tilde{r}_{\ell}=Q_{0}\left(X_{n+1}=\ell \mid X_{n}=\ell\right):=r_{\ell}, \ell \geq 1,
\end{aligned}
$$

and

$$
\tilde{p}_{0}=Q_{0}\left(X_{n+1}=1 \mid X_{n}=0\right):=p_{0}(S-1) /\left(S-p_{0}\right), \tilde{q}_{0}:=0, \tilde{r}_{0}:=r_{0} S /\left(S-p_{0}\right) .
$$

\section{Remark 2.2}

1. We suppose that the chain starts from zero only to simplify the computations, but it is possible to assume that the Markov chain $\left\{X_{n}\right\}_{n \geq 0}$ is starting from $x \neq 0$. One can obtain a similar result with minor modifications: for instance in (2.8) one needs to replace the factor $1 / S$ by $\left(\sum_{\ell \geq x} \gamma_{\ell}\right)^{-1}$.

2. As a main example of birth and death chain, we will consider (see also $\S 4.1$ below) the so-called Bessel random walk with index $\alpha>-1$ or of dimension $\delta=2(\alpha+1)>0$ (see also [7], p. 448, or [8]). The transition probabilities are given by

$$
p_{0}=1, \quad p_{\ell}=\frac{\ell+2 \alpha+1}{2 \ell+2 \alpha+1}, q_{\ell}=\frac{\ell}{2 \ell+2 \alpha+1}, r_{\ell}=0, \ell \geq 1 .
$$

If $\alpha=-1 / 2,\left\{X_{n}\right\}_{n \geq 0}$ is the absolute value of the symmetric standard random walk. Let us note that $\left\{X_{n}\right\}_{n \geq 0}$ is transient for $\alpha>0$, as we can easily see by using Proposition 2.1. 


\subsection{Continuous parameter setting}

Let $\{X(t)\}_{t \geq 0}$ be a Markov process parametrized by $t \in[0, \infty)$ and with the state space $\mathbb{N}$. For instance, we can think that $X(t)$ is the size of a population of particles at time $t$. We follow [10], p. 373, to describe the continuous time version of the birth and death process $\{X(t)\}_{t \geq 0}$. The changes of states yields as follows: when the process is in state $\ell$, assume that are given two independent random variables $B(\ell)$ and $D(\ell)$, which are independent from $\{X(t)\}_{t \geq 0}$, and exponentially distributed with parameters $b_{\ell}$ and $d_{\ell}$, respectively. A transition from $\ell$ to $\ell+1$ is made if $B(\ell) \leq D(\ell)$, which occurs with probability

$$
\mathbb{P}[B(\ell) \leq D(\ell)]=b_{\ell} /\left(b_{\ell}+d_{\ell}\right) ;
$$

otherwise a transition from $\ell$ to $\ell-1$ is made. The holding time in state $\ell$ is $B(\ell) \wedge D(\ell)$, which is exponential with parameter $b_{\ell}+d_{\ell}$ (we assume that $d_{0}=0$ ). We may think of $B(\ell)$ as the time until a birth when the population is $\ell$ and similarly, $D(\ell)$ is the time until a death when the population is $\ell$; the population increases by one if a birth occurs prior to a death; otherwise the population decreases by one.

Usually, the birth and death process in continuous time is described in terms of birth and death rates : in a population of size $\ell$ a particle is born at rate $b_{\ell}$ and dies at rate $d_{\ell}$. These refer to the infinitesimal transition probabilities:

$$
\mathbb{P}(X(t+h)=\ell+k \mid X(t)=\ell)=\left\{\begin{array}{ll}
b_{\ell} h+o(h), & \text { if } k=1 \\
d_{\ell} h+o(h), & \text { if } k=-1 \\
o(h), & \text { if }|k|>1,
\end{array} \quad \text { as } h \rightarrow 0 .\right.
$$

It can be seen that these assumptions lead to a model of jump Markov process with the holding time parameter at $\ell$

$$
\lambda_{\ell}:=b_{\ell}+d_{\ell}
$$

and the jump from $\ell$

$$
\xi_{\ell}=\left\{\begin{array}{ll}
+1 & \text { with probability } b_{\ell} /\left(b_{\ell}+d_{\ell}\right) \\
-1 & \text { with probability } d_{\ell} /\left(b_{\ell}+d_{\ell}\right)
\end{array} .\right.
$$

We will denote

$$
J_{0}=0 \quad \text { and, for } n \geq 1, \quad J_{n}=\inf \left\{t \geq J_{n-1}: X(t) \neq X\left(J_{n-1}\right)\right\} .
$$

Let us introduce the sequence of random variables $\left\{Y_{n}\right\}_{n \geq 0}$, given by

$$
Y_{n}:=X\left(J_{n}\right), n \geq 0 .
$$

The proof of the following proposition is classical (see, for instance [6], p. 76-77 and [9], p. 115):

\section{Proposition 2.3}

1. $\left\{Y_{n}\right\}_{n \geq 0}$ given by (2.16) is a birth and death discrete Markov chain with transition probabilities given by $p_{\ell}=b_{\ell} /\left(b_{\ell}+d_{\ell}\right), q_{\ell}=d_{\ell} /\left(b_{\ell}+d_{\ell}\right), \ell \geq 1$ and $p_{0}=1$. We set as previously

$$
\gamma_{0}=1 \quad \text { and } \quad \gamma_{\ell}=\prod_{k=1}^{\ell} \frac{q_{k}}{p_{k}}=\prod_{k=1}^{\ell} \frac{d_{k}}{b_{k}}, \ell \geq 1 .
$$


2. The continuous time birth and death process $\{X(t)\}_{t \geq 0}$ is transient if and only if the discrete Markov chain $\left\{Y_{n}\right\}_{n \geq 0}$ is transient, that is, if and only if

$$
S:=\sum_{\ell \geq 0} \gamma_{\ell}=1+\sum_{\ell \geq 1} \prod_{k=1}^{\ell} \frac{d_{k}}{b_{k}}<\infty .
$$

Let us denote

$$
V(t):=\int_{0}^{t} \mathbb{1}_{\{X(s)=0\}} d s, 0 \leq t \leq \infty .
$$

The second main result is:

Theorem 2.2 Let $\left\{\mathcal{F}_{t}\right\}_{t \geq 0}$ be the natural filtration associated to the transient birth and death process $\{X(t)\}_{t \geq 0}$ on the canonical probability space $\left(\Omega, \mathcal{F}_{\infty}, \mathbb{P}\right)$.

1. For any $t \geq 0$ and any event $A_{t} \in \mathcal{F}_{t}$,

$$
\lim _{\kappa \rightarrow \infty} \frac{\mathbb{E}_{0}\left[\mathbb{1}_{A_{t}} \mathbb{1}_{\{V(\infty) \geq \kappa\}}\right]}{\mathbb{E}_{0}\left[\mathbb{1}_{\{V(\infty) \geq \kappa\}}\right]}=\mathbb{E}_{0}\left[\mathbb{1}_{A_{t}} M(t)\right]
$$

where

$$
M(t):=\left(\frac{1}{S}\right) \exp \left(\frac{b_{0} V(t)}{S}\right)\left(\sum_{\ell \geq X(t)} \gamma_{\ell}\right) .
$$

2. $\{M(t)\}_{t \geq 0}$ is a positive $\left\{\mathcal{F}_{t}\right\}$-martingale which tends toward zero, almost surely. Henceforth $\{M(t)\}_{t \geq 0}$ is not uniformly integrable.

3. The equality (2.19) induces a probability measure $Q_{0}$ on $\left(\Omega, \mathcal{F}_{\infty}\right)$ : for any $t \geq 0$ and any event $A_{t} \in \mathcal{F}_{t}$

$$
Q_{0}\left(A_{t}\right):=\mathbb{E}_{0}\left[\mathbb{1}_{A_{t}} M(t)\right] .
$$

Then, under $Q_{0},\{X(t)\}_{t \geq 0}$ is a continuous recurrent birth and death process with birth and death rates given by:

$$
\tilde{b}_{\ell}:=b_{\ell}\left(\sum_{k \geq \ell+1} \gamma_{k}\right) /\left(\sum_{k \geq \ell} \gamma_{k}\right), \ell \geq 0, \tilde{d}_{\ell}:=d_{\ell}\left(\sum_{k \geq \ell-1} \gamma_{k}\right) /\left(\sum_{k \geq \ell} \gamma_{k}\right), \ell>0 .
$$

\subsection{Bessel random walk as a penalized classical random walk}

In this section we try to make the reverse work, more precisely, to penalize a recurrent birth and death Markov chain in order to obtain a transient birth and death Markov chain. We present a particular example of birth and death chain but the method of proof we have used in this case (see $\S 3.3$ below) seems more complicated for the general situation. Thus it remains an open question.

Let $p \in(0,1 / 2)$ and we consider a recurrent random walk $\left\{Z_{n}\right\}_{n \geq 0}$ with transition probabilities

$$
\begin{array}{r}
p_{\ell}:=\mathbb{P}\left(Z_{n+1}=\ell+1 \mid Z_{n}=\ell\right)=p, q_{\ell}:=\mathbb{P}\left(Z_{n+1}=\ell-1 \mid Z_{n}=\ell\right)=q=1-p, \forall \ell \geq 1 \\
\text { and } p_{0}=\mathbb{P}\left(Z_{n+1}=1 \mid Z_{n}=0\right)=1 .
\end{array}
$$

It is a recurrent Markov chain by Proposition 2.1, since $\sum_{\ell \geq 1} \gamma_{\ell}=\sum_{\ell \geq 1}(q / p)^{\ell}=\infty$. 
We shall penalize this chain to reach the state zero in an infinite time. As usual, we denote by $T_{0}$ the hitting time of state zero by the Markov chain $\left\{Z_{n}\right\}_{n \geq 0}$ and it is not very difficult to get an equivalent for $\mathbb{P}_{x}\left(T_{0} \geq \kappa\right)$, as $\kappa \rightarrow \infty$ (see Lemma 3.5 below). With this tool in hand, we can prove:

Proposition 2.4 Let $\left\{\mathcal{F}_{n}\right\}_{n \geq 0}$ be the natural filtration associated to the recurrent birth and death chain $\left\{Z_{n}\right\}_{n \geq 0}$ with the transition probabilities given by (2.23).

1. For any integers $x \geq 1, n \geq 0$ and any event $A_{n} \in \mathcal{F}_{n}$,

$$
\lim _{\kappa \rightarrow \infty} \frac{\mathbb{E}_{x}\left[\mathbb{1}_{A_{n}} \mathbb{1}_{\left\{T_{0} \geq \kappa\right\}}\right]}{\mathbb{E}_{x}\left[\mathbb{1}_{\left\{T_{0} \geq \kappa\right\}}\right]}=\mathbb{E}_{x}\left[\mathbb{1}_{A_{n}} M_{n}^{\prime}\right],
$$

where

$$
M_{n}^{\prime}:=\mathbb{1}_{\left\{T_{0}>n\right\}} \frac{Z_{n}}{x}\left(\frac{q}{p}\right)^{\left(Z_{n}-x\right) / 2}(4 p q)^{-n / 2}
$$

is a positive $\left\{\mathcal{F}_{n}\right\}$-martingale which tends toward zero, almost surely.

2. The equality (2.24) induces a probability measure $Q_{x}$ on $\left(\Omega, \mathcal{F}_{\infty}\right)$ : for any integer $n \geq 0$ and any event $A_{n} \in \mathcal{F}_{n}$,

$$
Q_{x}\left(A_{n}\right):=\mathbb{E}_{x}\left[\mathbb{1}_{A_{n}} M_{n}^{\prime}\right] .
$$

Then, under $Q_{x},\left\{Z_{n}\right\}_{n \geq 0}$ is a transient birth and death chain with transition probabilities given by:

$$
\begin{array}{r}
Q_{x}\left(Z_{n+1}=\ell+1 \mid Z_{n}=\ell\right)=\frac{\ell+1}{2 \ell}, Q_{x}\left(Z_{n+1}=\ell-1 \mid Z_{n}=\ell\right)=\frac{\ell-1}{2 \ell}, \quad \forall \ell \geq 1, \\
\text { and } Q_{x}\left(Z_{n+1}=0 \mid Z_{n}=1\right)=0, \quad(2.27)
\end{array}
$$

that is a Bessel random walk with index $1 / 2$ (or dimension 3).

Now, a very natural problem is to make a similar study for a recurrent birth and death process in continuous time setting. Consider a continuous time random walk $\{Z(t)\}_{t \geq 0}$ : when the process is in $\ell$ a transition in $\ell+1$ or in $\ell-1$ appears with probability $b /(b+d)$ or $d /(b+d)$, respectively. Here $b, d>0$ are the parameters of the exponential times until a birth or a death, respectively. Thus, the holding times in any state $\ell$ are exponential with parameter $b+d$. According to Proposition 2.3, the process $\{Z(t)\}_{t>0}$ is recurrent if and only if $d>b$.

It seems clearly that, if we penalize this process to reach the state zero in an infinite time, we obtain a transient Markov process, at least at a heuristic level. Moreover, in the light of Proposition 2.4, the underlying Markov chain should be a Bessel random walk with dimension 3. If $T_{0}^{\prime}$ denotes the hitting time of state zero by $\{Z(t)\}_{t \geq 0}$, it seems that the study of the equivalent of $\mathbb{P}_{x}\left(T_{0} \geq \kappa\right)$, as $\kappa \rightarrow \infty$, is more complicate as in the discrete time setting and it remains an open problem.

Let us point out that our intuition for the continuous time setting is reinforced by the study of the drifted recurrent Brownian motion. Indeed, we get the same phenomenon as in the discrete time setting : when one penalizes the process to reach 0 at an infinite time, the new process which we obtain is a Bessel process of dimension 3 . We state this result in the following: 
Proposition 2.5 Let $\{\bar{B}(t)\}_{t \geq 0}$ be a drifted Brownian motion $\bar{B}(t):=B(t)+\mu t$, where $\mu<0$ and $\{B(t)\}_{t \geq 0}$ is a standard Brownian motion starting from $x$. We denote, as usually, $\left\{\mathcal{F}_{t}^{(B, \mu)}\right\}_{t \geq 0}$ its natural filtration and $T_{0}^{(B, \mu)}$ the first hitting time of 0 by $\bar{B}$.

1. For any $t \geq 0$ and any $A_{t} \in \mathcal{F}_{t}^{(B, \mu)}$,

$$
\lim _{\kappa \rightarrow \infty} \frac{\mathbb{E}_{x}\left[\mathbb{1}_{A_{t}} \mathbb{1}_{\left\{T_{0}^{(B, \mu)}>\kappa\right\}}\right]}{\mathbb{E}_{x}\left[\mathbb{1}_{\left\{T_{0}^{(B, \mu)}>\kappa\right\}}\right]}=\mathbb{E}_{x}\left[\mathbb{1}_{A_{t}} M^{(B, \mu)}(t)\right],
$$

where

$$
M^{(B, \mu)}(t):=\mathbb{1}_{\left\{T_{0}^{(B, \mu)}>t\right\}}\left(\frac{\bar{B}(t)}{x}\right) \exp \left(-\mu(\bar{B}(t)-x)+\frac{t \mu^{2}}{2}\right)
$$

is a positive $\mathcal{F}^{(B, \mu)}$-martingale which tends toward zero, almost surely.

2. The equality (2.28) induces a probability measure $Q_{x}^{(B, \mu)}$ on: for any $t \geq 0$ and any $A_{t} \in \mathcal{F}_{t}^{(B, \mu)}$,

$$
Q_{x}^{(B, \mu)}\left(A_{t}\right):=\mathbb{E}_{x}\left[\mathbb{1}_{A_{t}} M^{(B, \mu)}(t)\right]
$$

and under $Q_{x}^{(B, \mu)}$, the infinitesimal generator of $\{\bar{B}(t)\}_{t \geq 0}$ is $\mathcal{L}=1 / 2 d^{2} / d x^{2}+1 / x d / d x$.

\section{Proofs}

\subsection{Proof of Theorem 2.1}

a. Proof of (2.7)-(2.8). To begin with, we split $\mathbb{E}_{0}\left[\mathbb{1}_{A_{n}} \mathbb{1}_{\left\{U_{\infty} \geq \kappa\right\}}\right]$ into two terms

$$
\begin{aligned}
\mathbb{E}_{0}\left[\mathbb{1}_{A_{n}} \mathbb{1}_{\left\{U_{\infty} \geq \kappa\right\}}\right]=\mathbb{E}_{0}\left[\mathbb{1}_{A_{n}}\left\{\mathbb{1}_{\left\{U_{n} \geq \kappa\right\}}+\mathbb{1}_{\left\{U_{\infty} \geq \kappa\right\}} \mathbb{1}_{\left\{U_{n}<\kappa\right\}}\right\}\right] & \\
& =\mathbb{E}_{0}\left[\mathbb{1}_{A_{n}} \mathbb{1}_{\left\{U_{n} \geq \kappa\right\}}\right]+\mathbb{E}_{0}\left[\mathbb{1}_{A_{n}} \mathbb{1}_{\left\{U_{\infty} \geq \kappa\right\}} \mathbb{1}_{\left\{U_{n}<\kappa\right\}}\right]
\end{aligned}
$$

and we note that $\mathbb{1}_{\left\{U_{n} \geq \kappa\right\}}$ vanishes if $\kappa \geq\lfloor n / 2\rfloor$.

Consider the second term on the right hand side of the latter equality and the Markov chain $\left\{\tilde{X}_{p}:=X_{n+p}-X_{n}\right\}_{p \geq 0}$. Then, for $\kappa \geq\lfloor n / 2\rfloor$,

$$
\begin{aligned}
\mathbb{E}_{0}\left[\mathbb{1}_{A_{n}} \mathbb{1}_{\left\{U_{\infty} \geq \kappa\right\}} \mathbb{1}_{\left\{U_{n}<\kappa\right\}}\right]=\mathbb{E}_{0}\left[\mathbb{1}_{A_{n}} \mathbb{1}_{\left\{U_{\infty} \geq \kappa\right\}}\right]= & \mathbb{E}_{0}\left[\mathbb{1}_{A_{n}} \mathbb{1}_{\left\{U_{\infty} \circ \theta_{n} \geq \kappa-U_{n}+\mathbb{1}_{\left\{X_{n}=0\right\}}\right\}}\right] \\
& =\mathbb{E}_{0}\left[\mathbb{1}_{A_{n}} \mathbb{E}_{X_{n}}\left(\mathbb{1}_{\left\{\tilde{U}_{\infty} \geq \kappa-U_{n}+\mathbb{1}_{\left\{X_{n}=0\right\}}\right\}}\right)\right] .
\end{aligned}
$$

Here we denoted $\tilde{U}_{\infty}=\sum_{p \geq 0} \mathbb{1}_{\left\{\tilde{X}_{p}=0\right\}}$ and, as usually, $\left\{\theta_{n}\right\}_{n \geq 0}$ the family of shift operators. At this level we need the following:

Lemma 3.1 For all integers $n \geq 0$,

$$
\mathbb{P}_{n}\left(U_{\infty} \geq \kappa\right)=\left(R_{n} / S\right)\left(1-p_{0} / S\right)^{\kappa-1},
$$

where

$$
R_{0}=S \quad \text { and } \quad R_{n}:=\sum_{\ell \geq n} \gamma_{\ell}, \quad n \geq 1
$$


Let us postpone the proof of this lemma and we finish the proof of (2.7). We deduce that, for $\kappa \geq\lfloor n / 2\rfloor$ :

$$
\mathbb{E}_{0}\left[\mathbb{1}_{A_{n}} \mathbb{1}_{\left\{U_{\infty} \geq \kappa\right\}}\right]=\mathbb{E}_{0}\left[\mathbb{1}_{A_{n}}\left(R_{X_{n}} / S\right)\left(1-p_{0} / S\right)^{\kappa-U_{n}+\mathbb{1}_{\left\{X_{n}=0\right\}}-1}\right]
$$

and, when we combine with (3.1) for $n=0$, we obtain, for $\kappa \geq\lfloor n / 2\rfloor$,

$$
\frac{\mathbb{E}_{0}\left[\mathbb{1}_{A_{n}} \mathbb{1}_{\left\{U_{\infty} \geq \kappa\right\}}\right]}{\mathbb{E}_{0}\left[\mathbb{1}_{\left\{U_{\infty} \geq \kappa\right\}}\right]}=\mathbb{E}_{0}\left[\mathbb{1}_{A_{n}}\left(R_{X_{n}} / S\right)\left(1-p_{0} / S\right)^{-U_{n}+\mathbb{1}_{\left\{X_{n}=0\right\}}}\right] .
$$

and the proof of the first part of Theorem 2.1 is done.

\section{Proof of Lemma 3.1.}

Let us consider $f: \mathbb{N} \rightarrow \mathbb{R}$ given by $f(0)=0$ and, for $n \geq 1$ integer, $f(n)=\sum_{\ell=0}^{n-1} \gamma_{\ell}$. Then, it is a direct computation to prove that $\left\{f\left(X_{n}\right): n \geq 0\right\}$ is a $\mathcal{F}_{n}$-martingale.

We denote by $T_{n}$ the hitting time of $n \in \mathbb{N}$ by the birth and death chain and we claim that, for all $n \geq 1$,

$$
\mathbb{P}_{n}\left(T_{0}<\infty\right)=\left(\sum_{\ell \geq n} \gamma_{\ell}\right) / S .
$$

Indeed, if $m \geq n$, by the optional stopping theorem, we get

$$
f(n)=\mathbb{E}_{n}\left[f\left(X_{T_{m} \wedge T_{0}}\right)\right]=f(m) \mathbb{P}_{n}\left(T_{m}<T_{0}\right)+f(n-1)\left[1-\mathbb{P}_{n}\left(T_{0}>T_{m}\right)\right]
$$

and we deduce

$$
\mathbb{P}_{n}\left(T_{m}<T_{0}\right)=(f(n)-f(0)) /(f(m)-f(0))=\left(\sum_{\ell=0}^{n-1} \gamma_{\ell}\right) /\left(\sum_{\ell=0}^{m-1} \gamma_{\ell}\right) .
$$

Since $\left\{X_{n}\right\}_{n \geq 0}$ is a transient Markov chain, by letting $m \rightarrow \infty$ we obtain

$$
\mathbb{P}_{n}\left(T_{0}=\infty\right)=\left(\sum_{\ell=0}^{n-1} \gamma_{\ell}\right) / S \quad \text { or } \quad \mathbb{P}_{n}\left(T_{0}<\infty\right)=\left(\sum_{\ell \geq n} \gamma_{\ell}\right) / S=R_{n} / S .
$$

Let us introduce the following stopping times :

$$
T_{0}^{(0)}=T_{0} \text { and } T_{0}^{(\ell)}:=\inf \left\{n \geq T_{0}^{(\ell-1)}: X_{n}=0\right\}, \text { if } \ell \geq 1 .
$$

By using (2.6), we can write

$$
\begin{aligned}
& \mathbb{P}_{0}\left(U_{\infty}=\ell\right)=\mathbb{P}_{0}\left(T_{0}^{(1)}+\ldots+T_{0}^{(\ell-1)}<\infty, T_{0}^{(1)}+\ldots+T_{0}^{(\ell)}=\infty\right) \\
&=\mathbb{P}_{0}\left(T_{0}^{(1)}<\infty\right) \mathbb{P}_{0}\left(T_{0}^{(2)}\right.\left.<\infty \mid T_{0}^{(1)}<\infty\right) \ldots \mathbb{P}_{0}\left(T_{0}^{(\ell-1)}<\infty \mid T_{0}^{(1)}+\ldots+T_{0}^{(\ell-1)}<\infty\right) \\
& \times \mathbb{P}_{0}\left(T_{0}^{(\ell)}=\infty \mid T_{0}^{(1)}+\ldots+T_{0}^{(\ell-1)}<\infty\right) \\
&= \mathbb{P}_{0}\left(T_{0}^{(1)}<\infty\right)^{\ell-1} \mathbb{P}_{0}\left(T_{0}^{(1)}=\infty\right)=\mathbb{P}_{1}\left(T_{0}<\infty\right)^{\ell-1} \mathbb{P}_{1}\left(T_{0}=\infty\right) \\
&=\left(p_{0} / S\right)\left(1-p_{0} / S\right)^{\ell-1} .
\end{aligned}
$$

Therefore, on the one hand

$$
\mathbb{P}_{0}\left(U_{\infty} \geq \kappa\right)=\sum_{\ell \geq \kappa} \mathbb{P}_{0}\left(U_{\infty}=\ell\right)=\left(1-p_{0} / S\right)^{\kappa-1},
$$

while, on the other hand, if $n \geq 1$,

$$
\mathbb{P}_{n}\left(U_{\infty} \geq \kappa\right)=\mathbb{P}_{n}\left(T_{0}<\infty\right) \mathbb{P}_{0}\left(U_{\infty} \geq \kappa\right)
$$

from which we get (3.1) and Lemma 3.1 is proved. 


\section{Remark 3.2}

Let us point out that in fact the limit in (2.7) is an exact equality for $\kappa>\lfloor n / 2\rfloor$. This is a somehow different situation with respect to those in [1] or [12].

b. Proof of the second part of Theorem 2.1. By using again the fact that $\left\{X_{n}\right\}_{n \geq 0}$ is a transient Markov chain, $\lim _{n \rightarrow \infty} X_{n}=\infty$ almost surely, and by using (2.1) the remainder of the series (2.6) tends to zero. Furthermore $U_{n}$ is almost surely finite, hence by (2.8) we obtain that $\lim _{n \rightarrow \infty} M_{n}=0$, almost surely. Then, necessarily, by assuming that $\left\{M_{n}\right\}_{n \geq 0}$ is a martingale, it can not be uniformly integrable, since $\mathbb{E}\left(M_{0}\right)=1$.

Hence the only thing we need to prove is that $\left\{M_{n}\right\}_{n \geq 0}$ is a martingale. Since, by (2.8) and (2.6), for each $n \geq 0$,

$$
0 \leq M_{n} \leq\left(1-p_{0} / S\right)^{-U_{n}} \leq\left(1-p_{0} / S\right)^{-n},
$$

we see that $M_{n}$ is integrable. We can write:

$$
\begin{array}{r}
\mathbb{E}\left[M_{n+1} \mid \mathcal{F}_{n}\right]=\mathbb{E}\left[M_{n+1} \mathbb{1}_{\left\{X_{n+1}=X_{n}+1\right\}} \mid \mathcal{F}_{n}\right]+\mathbb{E}\left[M_{n+1} \mathbb{1}_{\left\{X_{n+1}=X_{n-1} \mathbb{1}_{\left\{X_{n} \neq 0\right\}} \mid \mathcal{F}_{n}\right]}\right. \\
+\mathbb{E}\left[M_{n+1} \mathbb{1}_{\left\{X_{n+1}=X_{n}\right\}} \mid \mathcal{F}_{n}\right] .
\end{array}
$$

By using (2.8) and (3.2), the first term in (3.4) can be written as follows

$$
\begin{aligned}
& \mathbb{E}\left[(1 / S)\left(1-p_{0} / S\right)^{-U_{n}} R_{X_{n}+1} \mathbb{1}_{\left\{X_{n+1}=X_{n}+1\right\}} \mid \mathcal{F}_{n}\right] \\
& \quad=(1 / S)\left(1-p_{0} / S\right)^{-U_{n}} R_{X_{n}+1} \mathbb{E}\left[\mathbb{1}_{\left\{X_{n+1}=X_{n}+1\right\}} \mid \mathcal{F}_{n}\right]=(1 / S)\left(1-p_{0} / S\right)^{-U_{n}} R_{X_{n}+1} p_{X_{n}} .
\end{aligned}
$$

For the second term in (3.4) we split the expectation into two terms

$$
\begin{array}{r}
\mathbb{E}\left[M_{n+1} \mathbb{1}_{\left\{X_{n+1}=X_{n}-1\right\}} \mathbb{1}_{\left\{X_{n} \neq 0,1\right\}} \mid \mathcal{F}_{n}\right]+\mathbb{E}\left[M_{n+1} \mathbb{1}_{\left\{X_{n+1}=X_{n}-1\right\}} \mathbb{1}_{\left\{X_{n}=1\right\}} \mid \mathcal{F}_{n}\right] \\
=\mathbb{E}\left[(1 / S)\left(1-p_{0} / S\right)^{-U_{n}} R_{X_{n-1}-1} \mathbb{1}_{\left\{X_{n+1}=X_{n}-1\right\}} \mathbb{1}_{\left\{X_{n} \neq 0,1\right\}} \mid \mathcal{F}_{n}\right] \\
+\mathbb{E}\left[(1 / S)\left(1-p_{0} / S\right)^{-U_{n}-1+1} R_{X_{n-1}} \mathbb{1}_{\left\{X_{n+1}=X_{n}-1\right\}} \mathbb{1}_{\left\{X_{n}=1\right\}} \mid \mathcal{F}_{n}\right] \\
=(1 / S)\left(1-p_{0} / S\right)^{-U_{n}} R_{X_{n-1}} \mathbb{1}_{\left\{X_{n} \neq 0\right\}}\left(\mathbb{1}_{\left\{X_{n} \neq 1\right\}}+\mathbb{1}_{\left\{X_{n}=1\right\}}\right) \mathbb{E}\left[\mathbb{1}_{X_{n+1}=X_{n}-1} \mid \mathcal{F}_{n}\right] \\
=(1 / S)\left(1-p_{0} / S\right)^{-U_{n}} R_{X_{n-1}} \mathbb{1}_{\left\{X_{n} \neq 0\right\}} q_{X_{n}} .
\end{array}
$$

By a similar reasoning we can prove that the third term in (3.4) can be written

$$
\mathbb{E}\left[M_{n+1} \mathbb{1}_{\left\{X_{n+1}=X_{n}\right\}} \mid \mathcal{F}_{n}\right]=(1 / S)\left(1-p_{0} / S\right)^{-U_{n}} R_{X_{n}} r_{X_{n}} .
$$

We replace in (3.4) and we can write on the one hand,

$$
\begin{array}{r}
\mathbb{E}\left[M_{n+1} \mathbb{1}_{\left\{X_{n} \neq 0\right\}} \mid \mathcal{F}_{n}\right] \\
=(1 / S)\left(1-p_{0} / S\right)^{-U_{n}} \mathbb{1}_{\left\{X_{n} \neq 0\right\}}\left[p_{X_{n}} R_{X_{n}+1}+q_{X_{n}} R_{X_{n}-1}+r_{X_{n}} R_{X_{n}}\right] \\
=(1 / S)\left(1-p_{0} / S\right)^{-U_{n}} \mathbb{1}_{\left\{X_{n} \neq 0\right\}}\left[\left(p_{X_{n}}+q_{X_{n}}+r_{X_{n}}\right) R_{X_{n}}+q_{X_{n}} \gamma_{X_{n}-1}-p_{X_{n}} \gamma_{X_{n}}\right] \\
=M_{n} \mathbb{1}_{\left\{X_{n} \neq 0\right\}},
\end{array}
$$


by using (2.1), (2.3) and (3.2). On the other hand

$$
\begin{array}{r}
\mathbb{E}\left[M_{n+1} \mathbb{1}_{\left\{X_{n}=0\right\}} \mid \mathcal{F}_{n}\right]=(1 / S)\left(1-p_{0} / S\right)^{-U_{n}} \mathbb{1}_{\left\{X_{n}=0\right\}}\left\{p_{0} \sum_{\ell \geq 1} \gamma_{\ell}+r_{0} \sum_{\ell \geq 0} \gamma_{\ell}\right\} \\
=(1 / S)\left(1-p_{0} / S\right)^{-U_{n}} \mathbb{1}_{\left\{X_{n}=0\right\}} S\left(1-p_{0} / S\right)=(1 / S)\left(1-p_{0} / S\right)^{-U_{n}+1} \mathbb{1}_{\left\{X_{n}=0\right\}} \\
=M_{n} \mathbb{1}_{\left\{X_{n}=0\right\}},
\end{array}
$$

where we used the fact that $\sum_{\ell \geq 1} \gamma_{\ell}=S-1$ and $r_{0}=1-p_{0}$. Hence $\left\{M_{n}\right\}_{n \geq 0}$ is a martingale.

c. Proof of the third part of Theorem 2.1. Assuming that, under $Q_{0}$, the sequence $\left\{X_{n}\right\}_{n \geq 0}$ is a birth and death Markov chain, with transition probabilities given by (2.10), it is no difficult to prove that, under $Q_{0}$, the chain is recurrent. Indeed, it suffices to use the result of Proposition 2.1. Set

$$
\tilde{\gamma}_{0}:=1, \tilde{\gamma}_{\ell}:=\prod_{k=1}^{\ell} \frac{\tilde{q}_{k}}{\tilde{p}_{k}}, \ell \geq 1 .
$$

Therefore, by using (2.3), (2.10) and (3.2), we can write

$$
\begin{aligned}
\sum_{\ell \geq 1} \tilde{\gamma}_{\ell}=\sum_{\ell \geq 1} \prod_{k=1}^{\ell} \frac{q_{k} R_{k-1}}{p_{k} R_{k+1}} & =\sum_{\ell \geq 1} \gamma_{\ell} \prod_{k=1}^{\ell} \frac{R_{k-1}}{R_{k+1}}=\sum_{\ell \geq 1} \gamma_{\ell} \frac{R_{0} R_{1}}{R_{\ell} R_{\ell+1}} \\
& =S R_{1} \sum_{\ell \geq 1}\left(1 / R_{\ell+1}-1 / R_{\ell}\right)=S R_{1} \lim _{N \rightarrow \infty}\left(1 / R_{N+1}-1 / R_{1}\right)=\infty
\end{aligned}
$$

since $\lim _{N \rightarrow \infty} R_{N+1}=0$, by Proposition 2.1.

We prove that, under $Q_{0},\left\{X_{n}\right\}_{n \geq 0}$ is a birth and death Markov chain with transition probabilities given by (2.10). Consider, for all integers $n \geq 1$ and all integers $a_{0}, \ldots, a_{n-1} \geq 0$, the event

$$
A_{n-1}:=\left\{X_{0}=a_{0}, \ldots, X_{n-1}=a_{n-1}\right\} .
$$

We can write, for $\ell \geq 1$ and all integers $n \geq 1$,

$$
\begin{gathered}
\tilde{p}_{\ell}=Q_{0}\left[X_{n+1}=\ell+1 \mid X_{n}=\ell, A_{n-1}\right]=\frac{\mathbb{E}_{0}\left[\mathbb{1}_{\left\{X_{n+1}=\ell+1, X_{n}=\ell\right\} \cap A_{n-1}} M_{n+1}\right]}{\mathbb{E}_{0}\left[\mathbb{1}_{\left\{X_{n}=\ell\right\} \cap A_{n-1}} M_{n}\right]}, \quad \text { by }(2.9) \\
=\frac{\mathbb{E}_{0}\left[\mathbb{1}_{\left\{X_{n+1}=\ell+1, X_{n}=\ell\right\} \cap A_{n-1}}\left(1-p_{0} / S\right)^{\left.-U_{n+1} R_{\ell+1}\right]}\right.}{\mathbb{E}_{0}\left[\mathbb{1}_{\left\{X_{n}=\ell\right\} \cap A_{n-1}}\left(1-p_{0} / S\right)^{-U_{n}} R_{\ell}\right]}, \quad \text { by }(2.8) \\
=\frac{R_{\ell+1}}{R_{\ell}} \frac{\mathbb{E}_{0}\left[\mathbb { E } _ { 0 } \left[\mathbb{1}_{\left\{X_{n+1}=\ell+1, X_{n}=\ell\right\} \cap A_{n-1}}\left(1-p_{0} / S\right)^{\left.\left.-U_{n+1} \mid \mathcal{F}_{n}\right]\right]}\right.\right.}{\mathbb{E}_{0}\left[\mathbb{1}_{\left\{X_{n}=\ell\right\} \cap A_{n-1}}\left(1-p_{0} / S\right)^{-U_{n}}\right]} \\
=\frac{R_{\ell+1}}{R_{\ell}} \frac{\mathbb{E}_{0}\left[\mathbb{1}_{\left\{X_{n}=\ell\right\} \cap A_{n-1}}\left(1-p_{0} / S\right)^{-U_{n}} \mathbb{P}_{0}\left(X_{n+1}=X_{n}+1 \mid \mathcal{F}_{n}\right)\right]}{\mathbb{E}_{0}\left[\mathbb{1}_{\left\{X_{n}=\ell\right\} \cap A_{n-1}}\left(1-p_{0} / S\right)^{-U_{n}}\right]}, \quad \text { since } \ell \geq 1 \\
=\frac{R_{\ell+1}}{R_{\ell}} \frac{\mathbb{E}_{0}\left[\mathbb{1}_{\left\{X_{n}=\ell\right\} \cap A_{n-1}}\left(1-p_{0} / S\right)^{-U_{n}} p_{X_{n}}\right]}{\mathbb{E}_{0}\left[\mathbb{1}_{\left\{X_{n}=\ell\right\} \cap A_{n-1}}\left(1-p_{0} / S\right)^{-U_{n}}\right]}=p_{\ell} \frac{R_{\ell+1}}{R_{\ell} .}
\end{gathered}
$$


With a similar computation we get that, for $\ell \geq 1, \tilde{r}_{\ell}=r_{\ell}$.

Assume now that $\ell=0$. Then, by using a similar reasoning, for all $a_{0}, \ldots, a_{n-1} \geq 0$,

$$
\begin{aligned}
& \tilde{p}_{0}=Q_{0}\left[X_{n+1}=1 \mid X_{n}=0, A_{n-1}\right] \\
& \begin{aligned}
=\frac{\mathbb{E}_{0}\left[\mathbb{1}_{\left\{X_{n+1}=1, X_{n}=0\right\} \cap A_{n-1}}\left(1-p_{0} / S\right)^{-U_{n+1}} R_{1}\right]}{\mathbb{E}_{0}\left[\mathbb{1}_{\left\{X_{n}=0\right\} \cap A_{n-1}}\left(1-p_{0} / S\right)^{\left.-U_{n}+\mathbb{1}_{\left\{X_{n}=0\right\}} R_{0}\right]}\right.}, \quad \text { by }(2.9) \text { and }(2.8) \\
\quad=\frac{R_{1}}{S} \frac{\mathbb{E}_{0}\left[\mathbb{1}_{\left\{X_{n}=0\right\} \cap A_{n-1}}\left(1-p_{0} / S\right)^{-U_{n}} p_{X_{n}}\right]}{\mathbb{E}_{0}\left[\mathbb{1}_{\left\{X_{n}=0\right\} \cap A_{n-1}}\left(1-p_{0} / S\right)^{-U_{n}+1}\right]}=p_{0} \frac{R_{1}}{S}\left(1-\frac{p_{0}}{S}\right)^{-1} .
\end{aligned}
\end{aligned}
$$

Furthermore:

$$
\begin{aligned}
\tilde{r}_{0}=Q_{0}\left[X_{n+1}=\right. & \left.0 \mid X_{n}=0, A_{n-1}\right] \\
= & \frac{\mathbb{E}_{0}\left[\mathbb{1}_{\left\{X_{n+1}=0, X_{n}=0\right\} \cap A_{n-1}}\left(1-p_{0} / S\right)^{\left.-U_{n+1}+\mathbb{1}_{\left\{X_{n+1}=0\right\}} R_{0}\right]}\right.}{\mathbb{E}_{0}\left[\mathbb{1}_{\left\{X_{n}=0\right\} \cap A_{n-1}}\left(1-p_{0} / S\right)^{\left.-U_{n}+\mathbb{1}_{\left\{X_{n}=0\right\}} R_{0}\right]}\right.} \\
& =\frac{\mathbb{E}_{0}\left[\mathbb{1}_{\left\{X_{n+1}=0, X_{n}=0\right\} \cap A_{n-1}}\left(1-p_{0} / S\right)^{-U_{n}-1+1}\right]}{\mathbb{E}_{0}\left[\mathbb{1}_{\left\{X_{n}=0\right\} \cap A_{n-1}}\left(1-p_{0} / S\right)^{-U_{n}+1}\right]}=r_{0}\left(1-\frac{p_{0}}{S}\right)^{-1} .
\end{aligned}
$$

\subsection{Proof of Theorem 2.2}

The proof contains several similar ideas as in the proof of Theorem 2.1. We shall emphasize the main differences.

a. Proof of (2.19)-(2.20). This part is quite similar to the discrete case. We split

$$
\mathbb{E}_{0}\left[\mathbb{1}_{A_{t}} \mathbb{1}_{\{V(\infty) \geq \kappa\}}\right]=\mathbb{E}_{0}\left[\mathbb{1}_{A_{t}} \mathbb{1}_{\{V(t) \geq \kappa\}}\right]+\mathbb{E}_{0}\left[\mathbb{1}_{A_{t}} \mathbb{1}_{\{V(\infty) \geq \kappa\}} \mathbb{1}_{\{V(t)<\kappa\}}\right]
$$

and we note that $\mathbb{1}_{\{V(t) \geq \kappa\}}$ vanishes, if $\kappa>t$. Set $\{\tilde{X}(s):=X(t+s)-X(t)\}_{s \geq 0}$ and note that $V(\infty)=V(t)+V(\infty) \circ \theta_{t}$. Again and, as usually, $\left\{\theta_{t}\right\}_{t \geq 0}$ denotes the family of shift operators. Then, for $\kappa>t$ :

$$
\mathbb{E}_{0}\left[\mathbb{1}_{A_{t}} \mathbb{1}_{\{V(\infty) \geq \kappa\}} \mathbb{1}_{\{V(t)<\kappa\}}\right]=\mathbb{E}_{0}\left[\mathbb{1}_{A_{t}} \mathbb{1}_{\left\{V(\infty) \circ \theta_{t} \geq \kappa-V(t)\right\}}\right]=\mathbb{E}_{0}\left[\mathbb{1}_{A_{t}} \mathbb{E}_{X(t)}\left(\mathbb{1}_{\{\tilde{V}(\infty) \geq \kappa-V(t)}\right)\right],
$$

where $\tilde{V}(\infty):=\int_{0}^{\infty} \mathbb{1}_{\{\tilde{X}(t)=0\}} d s$. As for the discrete case we can state:

Lemma 3.3 For all integers $n \geq 0$, and for any $t \geq 0$,

$$
\mathbb{P}_{n}(V(\infty)>t)=\left(R_{n} / S\right) e^{-b_{0} t / S},
$$

where $R_{n}$ is given by (3.2).

Let us finish the proof of (2.19). For $\kappa>t$ :

$$
\mathbb{E}_{0}\left[\mathbb{1}_{A_{t}} \mathbb{1}_{\{V(\infty) \geq \kappa\}}\right]=\mathbb{E}_{0}\left[\mathbb{1}_{A_{t}}\left(R_{X(t)} / S\right) e^{-b_{0}(t-V(t)) / S}\right]
$$




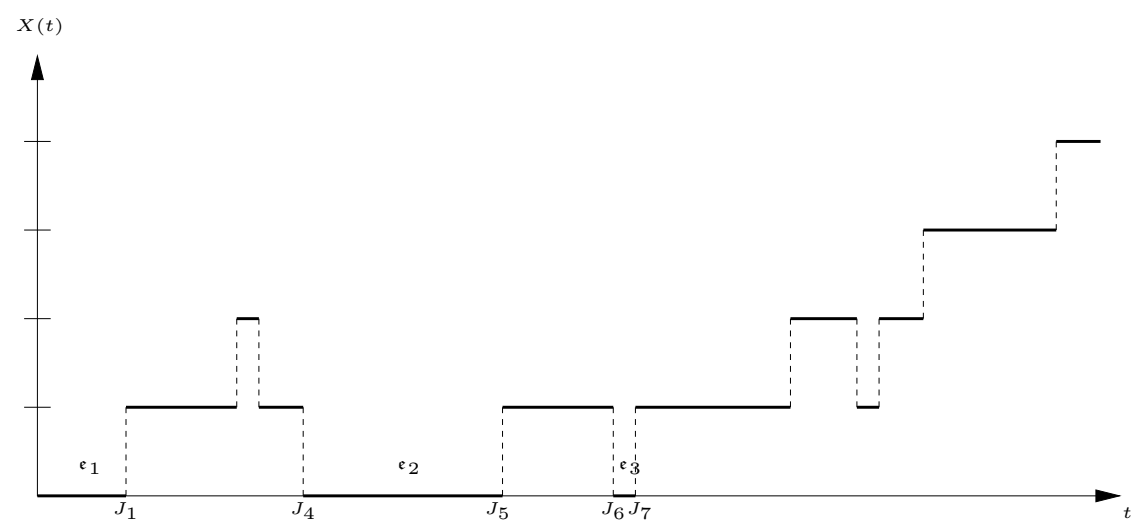

Figure 1: $X(t)$ conditionnally to the event $U_{\infty}=3$

and using the preceding lemma again, we obtain for $\kappa>t$ :

$$
\frac{\mathbb{E}_{0}\left[\mathbb{1}_{A_{t}} \mathbb{1}_{\{V(\infty) \geq \kappa\}}\right]}{\mathbb{E}_{0}\left[\mathbb{1}_{\{V(\infty) \geq \kappa\}}\right]}=\mathbb{E}_{0}\left[\mathbb{1}_{A_{t}}\left(R_{X(t)} / S\right) e^{b_{0} V(t) / S}\right]
$$

that is $(2.19)-(2.20)$.

Proof of Lemma 3.3. Recall that $\left\{Y_{n}\right\}_{n \geq 0}$ is given by (2.16) and we shall denote $U_{\infty}^{(Y)}:=$ $\sum_{n \geq 0} \mathbb{1}_{\left\{Y_{n}=0\right\}}$. It is no difficult to see that, on the event $\left\{U_{\infty}^{(Y)}=k\right\}, V(\infty)$ is the sum of $k$ independent random variables $\mathfrak{e}_{1}, \ldots, \mathfrak{e}_{k}$, the holding times at 0 , having exponential distribution of same parameter $\lambda_{0}=b_{0}$, and being independent from $U_{\infty}^{(Y)}$ (see Figure 1).

Therefore, by using (3.1), for all integers $n \geq 0$,

$$
\begin{aligned}
& \mathbb{P}_{n}(V(\infty)>t)=\sum_{k \geq 0} \mathbb{P}_{n}\left(U_{\infty}^{(Y)}=k, \mathfrak{e}_{1}+\ldots+\mathfrak{e}_{k}>t\right) \\
& =\sum_{k \geq 0} \mathbb{P}_{n}\left(U_{\infty}^{(Y)}=k\right) \mathbb{P}\left(\mathfrak{e}_{1}+\ldots+\mathfrak{e}_{k}>t\right)=\sum_{k \geq 0} \frac{R_{n}}{S}\left(1-\frac{1}{S}\right)^{k-1} \frac{1}{S} e^{-b_{0} t} \sum_{i=0}^{k-1} \frac{\left(b_{0} t\right)^{i}}{i !} \\
& =\frac{R_{n}}{S} \frac{1}{S} e^{-b_{0} t} \sum_{i \geq 0} \frac{\left(b_{0} t\right)^{i}}{i !} \sum_{k \geq i+1}\left(1-\frac{1}{S}\right)^{k-1}=\frac{R_{n}}{S} \frac{1}{S} e^{-b_{0} t} \sum_{i \geq 0} \frac{\left(b_{0} t\right)^{i}}{i !}\left(1-\frac{1}{S}\right)^{i} S=\frac{R_{n}}{S} e^{\frac{-b_{0} t}{S}}
\end{aligned}
$$

The proof of Lemma 3.3 is done.

\section{Remark 3.4}

We point out again that in fact the limit in (2.19) is an exact equality for $\kappa>t$, as was noted in the discrete parameter setting.

b. Proof of the second part of Theorem 2.2. As in the discrete case we point out a useful martingale. At this level we need to recall Dynkin's formula for pure jump processes 
(see, for instance [5], p. 262): if $f: \mathbb{N} \rightarrow \mathbb{R}$ is a bounded function such that $\{f(X(t))\}_{t \geq 0}$ is a pure jump process, then

$$
M^{f}(t):=f(X(t))-f(X(0))-\int_{0}^{t} L f(X(s)) d s
$$

is a local martingale, where we denoted

$$
L f(x):=b_{x}[f(x+1)-f(x)]+d_{x}[f(x-1)-f(x)] .
$$

If we take in (3.9) $f(x)=\sum_{\ell \geq x} \gamma_{\ell}=R_{x}$, we obtain:

$$
\begin{aligned}
& L f(x)=b_{x}\left(R_{x+1}-R_{x}\right)+d_{x}\left(R_{x-1}\right.\left.-R_{x}\right)=-b_{x} \gamma_{x}+d_{x} \gamma_{x-1} \\
&=-b_{x} \prod_{\ell=1}^{x} \frac{d_{\ell}}{b_{\ell}}+d_{x} \prod_{\ell=1}^{x-1} \frac{d_{\ell}}{b_{\ell}}=-b_{0} \gamma_{0} \mathbb{1}_{\{x=0\}}=-b_{0} \mathbb{1}_{\{x=0\}} .
\end{aligned}
$$

Hence $\left\{M^{f}(t)\right\}_{t \geq 0}$ given by (3.8) is a local martingale. Therefore, we can apply the integration by parts formula (see, for instance [5], p. 220) to the semimartingale

$$
R_{X(t)}=M^{f}(t)+S-\int_{0}^{t} b_{0} \mathbb{1}_{\{X(s)=0\}} d s
$$

and to the finite variation process $A(t):=\exp \left(b_{0} V(t) / S\right)$. We note that

$$
d R_{X(t)}=d M^{f}(t)-b_{0} \mathbb{1}_{\{X(t)=0\}} d t, \quad d A(t)=\left(b_{0} / S\right) e^{b_{0} V(t) / S} \mathbb{1}_{\{X(t)=0\}} d t
$$

and $\left\langle R_{X(\cdot)}, A\right\rangle=0$. We obtain, by using $(2.20)$,

$$
\begin{array}{r}
S M(t)=R_{X(t)} e^{b_{0} V(t) / S}=S+\int_{0}^{t} R_{X(s)} d A(s)+\int_{0}^{t} A(s) d R_{X(s)}+\left\langle R_{X(\cdot)}, A\right\rangle_{t} \\
=S+\int_{0}^{t} R_{X(s)} \frac{b_{0}}{S} e^{b_{0} V(s) / S} \mathbb{1}_{\{X(s)=0\}} d s+\int_{0}^{t} e^{b_{0} V(s) / S}\left(d M^{f}(s)-b_{0} \mathbb{1}_{\{X(s)=0\}} d s\right) \\
=S+\int_{0}^{t} e^{b_{0} V(s) / S} d M^{f}(s)
\end{array}
$$

and consequently, $\{M(t)\}_{t \geq 0}$ is a local martingale. In fact, it is a (true) martingale since, for all $T \geq 0, \mathbb{E}\left(\sup _{0 \leq t \leq T}|M(t)|\right) \leq \exp \left(b_{0} T / s\right)$.

b. Proof of the third part of Theorem 2.2. First, we prove that, under $Q_{0},\{X(t)\}_{t \geq 0}$ is a Markov process. For any $n \geq 1$ and arbitrary sequences $0 \leq t_{0}<t_{1}<\ldots<t_{n-1}$ and $a_{0}, \ldots, a_{n-1} \in \mathbb{N}$, we consider the event

$$
A_{n-1}=\left\{X\left(t_{0}\right)=a_{0}, \ldots, X\left(t_{n-1}\right)=a_{n-1}\right\} .
$$


Then, for all integers $k, \ell \geq 0$,

$$
\begin{gathered}
Q_{0}\left(X\left(t_{n+1}\right)=\ell \mid X\left(t_{n}\right)=k, A_{n-1}\right) \\
=\frac{\mathbb{E}_{0}\left[\mathbb{1}_{\left\{X\left(t_{n+1}\right)=\ell, X\left(t_{n}\right)=k\right\} \cap A_{n-1}} M\left(t_{n+1}\right)\right]}{\mathbb{E}_{0}\left[\mathbb{1}_{\left\{X\left(t_{n}\right)=k\right\} \cap A_{n-1}} M\left(t_{n}\right)\right]}=\frac{\mathbb{E}_{0}\left[\mathbb{1}_{\left.\left\{X\left(t_{n+1}\right)=\ell, X\left(t_{n}\right)=k\right\} \cap A_{n-1} R_{\ell} e^{b_{0} V\left(t_{n+1}\right) / S}\right]}\right.}{\mathbb{E}_{0}\left[\mathbb{1}_{\left\{X\left(t_{n}\right)=k\right\} \cap A_{n-1}} R_{k} e^{b_{0} V\left(t_{n}\right) / S}\right]} \\
=\frac{R_{\ell}}{R_{k}} \frac{\mathbb{E}_{0}\left[\mathbb{1}_{\left\{X\left(t_{n+1}\right)=\ell, X\left(t_{n}\right)=k\right\} \cap A_{n-1}} \exp \left(b_{0}\left(V\left(t_{n}\right)+V\left(t_{n+1}-t_{n}\right) \circ \theta_{t_{n}}\right) / S\right)\right]}{\mathbb{E}_{0}\left[\mathbb{1}_{\left\{X\left(t_{n}\right)=k\right\} \cap A_{n-1}} e^{b_{0} V\left(t_{n}\right) / S}\right]} \\
=\frac{R_{\ell}}{R_{k}} \frac{\mathbb{E}_{0}\left[\mathbb{1}_{\left\{X\left(t_{n}\right)=k\right\} \cap A_{n-1}} e^{b_{0} V\left(t_{n}\right) / S} \mathbb{E}\left[\mathbb{1}_{\left\{X\left(t_{n+1}\right)=\ell\right\}} \exp \left(b_{0}\left(V\left(t_{n+1}-t_{n}\right) \circ \theta_{t_{n}}\right) / S\right) \mid \mathcal{F}_{t_{n}}\right]\right]}{\mathbb{E}_{0}\left[\mathbb{1}_{\left\{X\left(t_{n}\right)=k\right\} \cap A_{n-1}} e^{b_{0} V\left(t_{n}\right) / S}\right]} \\
\mathbb{E}_{0}\left[\mathbb{1}_{\left\{X\left(t_{n}\right)=k\right\} \cap A_{n-1}} e^{b_{0} V\left(t_{n}\right) / S}\right] \\
=\frac{R_{\ell}}{R_{k}} \frac{\mathbb{E}_{0}\left[\mathbb{1}_{\left\{X\left(t_{n}\right)=k\right\} \cap A_{n-1}} e^{b_{0} V\left(t_{n}\right) / S} \mathbb{E}_{k}\left[\mathbb{1}_{\left\{\tilde{X}\left(t_{n+1}-t_{n}\right)=\ell\right\}} e^{b_{0} \tilde{V}\left(t_{n+1}-t_{n}\right) / S}\right]\right]}{R_{k}\left[\mathbb{1}_{\left\{\tilde{X}\left(t_{n+1}-t_{n}\right)=\ell\right\}} e^{b_{0} \tilde{V}\left(t_{n+1}-t_{n}\right) / S}\right],}
\end{gathered}
$$

which does not depend on $A_{n-1}$. This proves that, under $Q_{0},\{X(t)\}_{t \geq 0}$ is a Markov process.

Let us study the holding times, under $Q_{0}$, of the Markov process $\{X(t)\}_{t \geq 0}$, for instance we shall compute the distribution of $J_{1}$. For any integer $\ell \geq 1$ and for any $\alpha \geq 0$ :

$$
\begin{gathered}
\mathbb{E}_{\ell}^{Q_{0}}\left[e^{-\alpha J_{1}}\right]=\frac{\mathbb{E}^{Q_{0}}\left[e^{-\alpha J_{1}} \mathbb{1}_{\{X(0)=\ell\}}\right]}{\mathbb{E}^{Q_{0}}[X(0)=\ell]}=\frac{\mathbb{E}\left[\mathbb{1}_{\{X(0)=\ell\}} e^{-\alpha J_{1}} M\left(J_{1}\right)\right]}{\mathbb{E}\left[\mathbb{1}_{\{X(0)=\ell\}} M(0)\right]}=\frac{S}{R_{\ell}} \mathbb{E}_{\ell}\left[e^{-\alpha J_{1}} M\left(J_{1}\right)\right] \\
=\frac{S}{R_{\ell}}\left(\mathbb{E}_{\ell}\left[\mathbb{1}_{\{B(\ell) \leq D(\ell)\}} e^{-\alpha B(\ell)} M(B(\ell))\right]+\mathbb{E}_{\ell}\left[\mathbb{1}_{\{D(\ell) \leq B(\ell)\}} e^{-\alpha D(\ell)} M(D(\ell))\right]\right) \\
=\frac{1}{R_{\ell}}\left(\mathbb{E}_{\ell}\left[\mathbb{1}_{\{B(\ell) \leq D(\ell)\}} e^{-\alpha B(\ell)} R_{\ell+1}\right]+\mathbb{E}_{\ell}\left[\mathbb{1}_{\{D(\ell) \leq B(\ell)\}} e^{-\alpha D(\ell)} R_{\ell-1}\right]\right),
\end{gathered}
$$

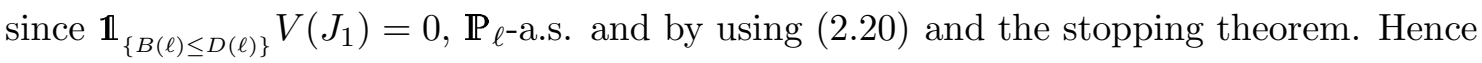

$$
\mathbb{E}_{\ell}^{Q_{0}}\left[e^{-\alpha J_{1}}\right]=\frac{R_{\ell+1}}{R_{\ell}} \mathbb{E}_{\ell}\left[\mathbb{1}_{\{B(\ell) \leq D(\ell)\}} e^{-\alpha B(\ell)}\right]+\frac{R_{\ell-1}}{R_{\ell}} \mathbb{E}_{\ell}\left[\mathbb{1}_{\{D(\ell) \leq B(\ell)\}} e^{-\alpha D(\ell)}\right] .
$$

By using (2.13) we can compute :

$$
\mathbb{E}_{\ell}\left[\mathbb{1}_{\{B(\ell) \leq D(\ell)\}} e^{-\alpha B(\ell)}\right]=\int_{0}^{\infty} d x e^{-\alpha x} e^{-b_{\ell} x} b_{\ell} \int_{x}^{\infty} d y e^{-d_{\ell} y} d_{\ell}=\frac{b_{\ell}}{b_{\ell}+d_{\ell}+\alpha} .
$$

Therefore, we obtain:

$$
\mathbb{E}_{\ell}^{Q_{0}}\left[e^{-\alpha J_{1}}\right]=\frac{b_{\ell} R_{\ell+1}+d_{\ell} R_{\ell-1}}{R_{\ell}\left(b_{\ell}+d_{\ell}+\alpha\right)}=\frac{b_{\ell}+d_{\ell}}{b_{\ell}+d_{\ell}+\alpha} .
$$

We conclude that the holding time at $\ell \geq 1$ is an exponential random variable with parameter $\lambda_{\ell}=b_{\ell}+d_{\ell}$. We stress that for $\ell \geq 1$ the distribution of the holding time is the same under the probabilities $\mathbb{P}$ and $Q_{0}$. 
The reasoning is similar for $\ell=0$, :

$$
\begin{aligned}
\mathbb{E}_{0}^{Q_{0}}\left[e^{-\alpha J_{1}}\right]=\frac{\mathbb{E}\left[\mathbb{1}_{\{X(0)=0\}} e^{-\alpha J_{1}} M\left(J_{1}\right)\right]}{\mathbb{E}\left[\mathbb{1}_{\{X(0)=0\}} M(0)\right]}=\mathbb{E}_{0}\left[e^{-\alpha B(0)} \frac{R_{1}}{S} e^{b_{0} V(B(0)) / S}\right] \\
=\frac{R_{1}}{S} \mathbb{E}_{0}\left[e^{-\alpha B(0)} e^{\frac{b_{0} B(0)}{S}}\right]=\frac{S-1}{S} \mathbb{E}_{0}\left[e^{B(0)\left(-\alpha+b_{0} / S\right)}\right]=\frac{b_{0}-b_{0} / S}{b_{0}-b_{0} / S+\alpha},
\end{aligned}
$$

and we deduce that the holding time at 0 is an exponential random variable of parameter $b_{0}(S-1) / S$.

Now, we study the underlying Markov chain $\left\{Y_{n}\right\}_{n \geq 0}$ under $Q_{0}$. For any positive Borel function $g: \mathbb{N} \rightarrow \mathbb{R}$ and $\ell \geq 1$, as previously:

$$
\begin{gathered}
\mathbb{E}_{\ell}^{Q_{0}}\left[g\left(Y_{1}\right)\right]=\mathbb{E}_{\ell}^{Q_{0}}\left[g\left(X\left(J_{1}\right)\right)\right]=\frac{\mathbb{E}^{Q_{0}}\left[\mathbb{1}_{\{X(0)=\ell\}} g\left(X\left(J_{1}\right)\right)\right]}{\mathbb{E}^{Q_{0}}\left[\mathbb{1}_{\{X(0)=\ell\}}\right]} \\
=\frac{\mathbb{E}\left[\mathbb{1}_{\{X(0)=\ell\}} \mathbb{1}_{\{B(\ell) \leq D(\ell)\}} g(\ell+1) R_{\ell+1}\right]}{\mathbb{E}^{Q_{0}}\left[\mathbb{1}_{\{X(0)=\ell\}} R_{\ell}\right]}+\frac{\mathbb{E}\left[\mathbb{1}_{\{X(0)=\ell\}} \mathbb{1}_{\{D(\ell) \leq B(\ell)\}} g(\ell-1) R_{\ell-1}\right]}{\mathbb{E}^{Q_{0}}\left[\mathbb{1}_{\{X(0)=\ell\}} R_{\ell}\right]} \\
=g(\ell+1) \frac{R_{\ell+1}}{R_{\ell}} \mathbb{E}_{\ell}\left[\mathbb{1}_{\{B(\ell) \leq D(\ell)\}}\right]+g(\ell-1) \frac{R_{\ell-1}}{R_{\ell}} \mathbb{E}_{\ell}\left[\mathbb{1}_{\{D(\ell) \leq B(\ell)\}}\right] \\
=g(\ell+1) \frac{R_{\ell+1}}{R_{\ell}} \frac{b_{\ell}}{b_{\ell}+d_{\ell}}+g(\ell-1) \frac{R_{\ell-1}}{R_{\ell}} \frac{d_{\ell}}{b_{\ell}+d_{\ell}}
\end{gathered}
$$

For $\ell=0$, the reasoning is the same. We conclude that under $Q_{0},\left\{Y_{n}\right\}_{n \geq 0}$ is a birth and death Markov chain with discrete parameter and the transition probabilities:

$$
\tilde{p}_{\ell}=\frac{R_{\ell+1}}{R_{\ell}} \frac{b_{\ell}}{b_{\ell}+d_{\ell}}, \quad \tilde{q}_{\ell}=\frac{R_{\ell-1}}{R_{\ell}} \frac{d_{\ell}}{b_{\ell}+d_{\ell}}, \text { for } \ell \geq 1, \quad \text { and } \tilde{p}_{0}=1 .
$$

Then if we set $\tilde{\gamma}_{0}=1$ and $\tilde{\gamma}_{\ell}=\prod_{i=1}^{\ell} \frac{\tilde{q}_{i}}{\tilde{p}_{i}}$, for $\ell \geq 1$, we have:

$$
\sum_{\ell \geq 0} \tilde{\gamma}_{\ell}=1+\sum_{\ell \geq 1} \prod_{i=1}^{\ell} \frac{d_{i} R_{i-1}}{b_{i} R_{i+1}}=1+\sum_{\ell \geq 1} \prod_{i=1}^{\ell} \frac{q_{i} R_{i-1}}{p_{i} R_{i+1}} .
$$

According to (3.6) and Proposition 2.1, $\left\{Y_{n}\right\}_{n \geq 0}$ is recurrent. Furthermore, according to the second point of the Proposition (2.3), $\{X(t)\}_{t \geq 0}$ is recurrent under $Q_{0}$.

If we denote $\tilde{b}_{\ell}$ and $\tilde{d}_{\ell}$ the birth and death rates of $(X(t), t \geq 0)$ under $Q_{0}$, we have:

$$
\tilde{b}_{\ell}+\tilde{d}_{\ell}=b_{\ell}+d_{\ell} \quad \text { and } \quad \tilde{p}_{\ell}=\frac{\tilde{b}_{\ell}}{\tilde{b}_{\ell}+\tilde{d}_{\ell}}=\frac{R_{\ell+1}}{R_{\ell}} \frac{b_{\ell}}{b_{\ell}+d_{\ell}} .
$$

Consequently,

$$
\tilde{b}_{\ell}=b_{\ell} \frac{R_{\ell+1}}{R_{\ell}} \quad \text { and } \quad \tilde{d}_{\ell}=d_{\ell} \frac{R_{\ell-1}}{R_{\ell}}
$$

and (2.22) is proved. 


\subsection{Proofs of Propositions 2.4 and 2.5}

Proof of Proposition 2.4. To begin with, we need to find an equivalent, as $\kappa \rightarrow \infty$, for $\mathbb{P}_{x}\left(T_{0} \geq \kappa\right)$, when $x \geq 1$ is an integer. For the sake of completeness we state this in the following:

Lemma 3.5 Let $x \geq 1$ an integer. As $\kappa \rightarrow \infty$,

$$
\mathbb{P}_{x}\left(T_{0} \geq \kappa\right) \sim 4\left(\frac{2}{\pi}\right)^{1 / 2} x\left(\frac{q}{p}\right)^{x / 2} \kappa^{-3 / 2}(4 p q)^{\kappa / 2}
$$

As previously, we postpone the proof of this lemma and we finish the proof of (2.24)-(2.25). As $\kappa \rightarrow \infty$, we obtain the following equivalent

$$
\begin{aligned}
\frac{\mathbb{E}_{x}\left[\mathbb{1}_{\left\{A_{n}, T_{0} \geq \kappa\right\}}\right]}{\mathbb{E}_{x}\left[\mathbb{1}_{\left\{T_{0} \geq \kappa\right\}}\right]}=\frac{\mathbb{E}_{x}\left[\mathbb{1}_{\left\{\Lambda_{n}, T_{0} \geq n\right\}} \mathbb{E}_{Z_{n}}\left[\mathbb{1}_{\widetilde{T}_{0} \geq \kappa-n}\right]\right]}{\mathbb{E}_{x}\left[\mathbb{1}_{\left\{T_{0} \geq \kappa\right\}}\right]} & \underset{\kappa \rightarrow \infty}{\sim} \frac{\mathbb{E}_{x}\left[\mathbb{1}_{\left\{A_{n}, T_{0} \geq n\right\}} 4\left(\frac{2}{\pi}\right)^{1 / 2} Z_{n}\left(\frac{q}{p}\right)^{Z_{n} / 2}(\kappa-n)^{-3 / 2}(4 p q)^{(\kappa-n) / 2}\right]}{4\left(\frac{2}{\pi}\right)^{1 / 2} x\left(\frac{q}{p}\right)^{x / 2} \kappa^{-3 / 2}(4 p q)^{\kappa / 2}}
\end{aligned}
$$

and we deduce the expression of $M_{n}^{\prime},(2.25)$. As usually, we verify the fact that it is a martingale. Firstly,

$$
\begin{array}{r}
\mathbb{E}_{x}\left[M_{n+1}^{\prime} \mathbb{1}_{\left\{Z_{n} \geq 2\right\}} \mid \mathcal{F}_{n}\right]=\mathbb{1}_{\left\{T_{0} \geq n, Z_{n} \geq 2\right\}} \frac{Z_{n}}{x}\left(\frac{q}{p}\right)^{\left(Z_{n}-x\right) / 2}(4 p q)^{-(n+1) / 2} \mathbb{E}\left[\left(\frac{q}{p}\right)^{\xi_{n+1 / 2}} \mid \mathcal{F}_{n}\right] \\
+\mathbb{1}_{\left\{T_{0} \geq n, Z_{n} \geq 2\right\}} \frac{1}{x}\left(\frac{q}{p}\right)^{\left(Z_{n}-x\right) / 2}(4 p q)^{-(n+1) / 2} \mathbb{E}\left[\xi_{n+1}\left(\frac{q}{p}\right)^{\xi_{n+1} / 2} \mid \mathcal{F}_{n}\right] \\
=\mathbb{1}_{\left\{T_{0} \geq n, Z_{n} \geq 2\right\}} \frac{Z_{n}}{x}\left(\frac{q}{p}\right)^{\left(Z_{n}-x\right) / 2}(4 p q)^{-(n+1) / 2}\left[p\left(\frac{q}{p}\right)^{1 / 2}+q\left(\frac{q}{p}\right)^{-1 / 2}\right] \\
+\mathbb{1}_{\left\{T_{0} \geq n, Z_{n} \geq 2\right\}} \frac{1}{x}\left(\frac{q}{p}\right)^{\left(Z_{n}-x\right) / 2}(4 p q)^{-(n+1) / 2}\left[p\left(\frac{q}{p}\right)^{1 / 2}-q\left(\frac{q}{p}\right)^{-1 / 2}\right]=\mathbb{1}_{\left\{Z_{n} \geq 2\right\}} M_{n}^{\prime},
\end{array}
$$

and, secondly,

$$
\begin{aligned}
\mathbb{E}_{x}\left[M_{n+1}^{\prime} \mathbb{1}_{\left\{Z_{n}=1\right\}} \mid \mathcal{F}_{n}\right]=\mathbb{E}_{x}\left[\mathbb{1}_{\left\{T_{0} \geq n Z_{n+1}=2, Z_{n}=1\right\}} \frac{2}{x}\left(\frac{q}{p}\right)^{(2-x) / 2}(4 p q)^{-(n+1) / 2}\right] \\
=\mathbb{1}_{\left\{T_{0} \geq n, Z_{n}=1\right\}} \frac{2}{x}\left(\frac{q}{p}\right)^{(1-x) / 2}(4 p q)^{-n / 2}\left(\frac{q}{p}\right)^{1 / 2}(4 p q)^{-1 / 2} p=\mathbb{1}_{\left\{Z_{n}=1\right\}} M_{n}^{\prime} .
\end{aligned}
$$


Finally, to verify $(2.27)$, we denote again $A_{n-1}:=\left\{Z_{0}=a_{0}, \ldots, Z_{n-1}=a_{n-1}\right\}$. If $\ell \geq 1$ :

$$
\begin{aligned}
& Q_{x}\left(Z_{n+1}=\right.\left.\ell+1 \mid Z_{n}=\ell, A_{n-1}\right)=\frac{\mathbb{E}_{x}\left[\mathbb{1}_{\left.\left\{Z_{n+1}=\ell+1, Z_{n}=\ell\right\} \cap A_{n-1} M_{n+1}\right]}\right.}{\mathbb{E}_{x}\left[\mathbb{1}_{\left\{Z_{n}=\ell\right\} \cap A_{n-1}} M_{n}^{\prime}\right]} \\
&= \frac{\mathbb{E}_{x}\left[\mathbb{1}_{\left\{T_{0} \geq n+1, Z_{n+1}=\ell+1, Z_{n}=\ell,\right\} \cap A_{n-1}} \frac{\ell+1}{x}\left(\frac{q}{p}\right)^{(\ell+1-x) / 2}(4 p q)^{-(n+1) / 2}\right]}{\mathbb{E}_{x}\left[\mathbb{1}_{\left.\left\{T_{0} \geq n, Z_{n}=\ell,\right\} \cap A_{n-1} \frac{\ell}{x}\left(\frac{q}{p}\right)^{(\ell-x) / 2}(4 p q)^{-n / 2}\right]}\right]} \\
&= \frac{\ell+1}{\ell}\left(\frac{q}{p}\right)^{1 / 2}(4 p q)^{-1 / 2} \frac{\mathbb{E}_{x}\left[\mathbb{1}_{\left\{T_{0} \geq n, Z_{n+1}=\ell+1, Z_{n}=\ell\right\} \cap A_{n-1}}\right]}{\mathbb{E}_{x}\left[\mathbb{1}_{\left.\left\{T_{0} \geq n, Z_{n}=\ell\right\} \cap A_{n-1}\right]}\right]}=\frac{\ell+1}{2 \ell p} \\
& \times \mathbb{P}_{x}\left(T_{0} \geq n, Z_{n+1}=\ell+1 \mid Z_{n}=\ell, A_{n-1}\right)=\frac{\ell+1}{2 \ell p} \mathbb{P}\left(Z_{n+1}=\ell+1 \mid Z_{n}=\ell\right)=\frac{\ell+1}{2 \ell},
\end{aligned}
$$

where we used the Markovian feature of $\left\{Z_{n}\right\}$ under $\mathbb{P}_{x}$. Similarly, we show that

$$
Q_{x}\left(Z_{n+1}=\ell-1 \mid Z_{n}=\ell\right)=\frac{\ell-1}{2 \ell} .
$$

Also, it is clear that $Q_{x}\left(Z_{n+1}=0 \mid Z_{n}=1\right)=0$ and (2.27) is proved.

Proof of Lemma 3.5. To begin with, recall that (see for instance [2], p. 351), for all $s \in[0,1]$

$$
\mathbb{E}_{x}\left(s^{T_{0}}\right)=\left(\frac{1-\sqrt{1-4 p q s^{2}}}{2 p s}\right)^{x}=\sum_{r \geq x} \mathbb{P}_{x}\left(T_{0}=r\right) s^{r}
$$

It is a direct computation to get:

$$
\left(\frac{1-\sqrt{1-4 p q s^{2}}}{2 p s}\right)^{x}=\frac{1}{(2 p)^{x}} \sum_{\ell \geq x} \frac{(-4 p q)^{\ell}}{\ell !} s^{2 \ell-x} \sum_{k=0}^{x}(-1)^{k}\left(\begin{array}{l}
x \\
k
\end{array}\right) \prod_{i=0}^{\ell-1}\left(\frac{k}{2}-i\right) .
$$

Therefore, if $x$ and $r$ are of same evenness we find

$$
\begin{aligned}
P_{x}\left(T_{0}=r\right)=\frac{(-4 p q)^{(n+r) / 2}}{(2 p)^{x}((x+r) / 2) !} \sum_{k=0}^{x}(-1)^{k}\left(\begin{array}{l}
x \\
k
\end{array}\right) \prod_{i=0}^{(x+r) / 2-1}\left(\frac{k}{2}-i\right) \\
=-\frac{(-4 p q)^{(x+r) / 2}}{(2 p)^{x}\left(\frac{(x+r)}{2}\right) !} \sum_{k^{\prime}=0}^{\left\lfloor\frac{x-1}{2}\right\rfloor}\left(\begin{array}{c}
x \\
2 k^{\prime}+1
\end{array}\right) \prod_{i=0}^{\frac{(x+r)}{2}-1}\left(k^{\prime}-i+\frac{1}{2}\right) .
\end{aligned}
$$

After some algebraic computations we obtain

$$
\mathbb{P}_{x}\left(T_{0}=r\right)=\frac{(4 p q)^{(x+r) / 2}}{\pi(2 p)^{x}((x+r) / 2) !} \sum_{k^{\prime}=0}^{\left\lfloor\frac{x-1}{2}\right\rfloor}(-1)^{k^{\prime}}\left(\begin{array}{c}
x \\
2 k^{\prime}+1
\end{array}\right) \Gamma\left(k^{\prime}+\frac{3}{2}\right) \Gamma\left(\frac{x+r}{2}-k^{\prime}-\frac{1}{2}\right) .
$$

As $r \rightarrow \infty$, the only significant term in the latter sum corresponds to $k^{\prime}=0$, hence

$$
\mathbb{P}_{x}\left(T_{0}=r\right) \underset{r \rightarrow \infty}{\sim} \frac{x \Gamma(3 / 2)}{\pi\left(\frac{x+r}{2}\right) !}\left(\frac{q}{p}\right)^{x / 2}(4 p q)^{\frac{r}{2}} \Gamma\left(\frac{x+r}{2}-\frac{1}{2}\right) \sim \frac{2 x}{\sqrt{\pi}}\left(\frac{q}{p}\right)^{x / 2}\left(\frac{r}{2}\right)^{-3 / 2}(4 p q)^{r / 2}
$$


Finally, since $\mathbb{P}_{x}\left(T_{0} \geq \kappa\right)=\sum_{r \geq \kappa} \mathbb{P}_{x}\left(T_{0}=r\right)$, it is no difficult to prove that, as $\kappa \rightarrow \infty$, $\mathbb{P}_{x}\left(T_{0} \geq \kappa\right) \sim \mathbb{P}_{x}\left(T_{0}=\kappa\right)$, the first term again. The proof of (3.10) is done.

Proof of Propositions 2.5. It is a classical result, using the Girsanov theorem (see, for instance [4], p. 197), that, for $x>0$ :

$$
\mathbb{P}_{x}\left(T_{0}^{(B, \mu)}>\kappa\right)=\mathbb{P}_{0}\left(T_{-x}^{(B, \mu)}>\kappa\right)=\int_{\kappa}^{\infty} \frac{x}{\sqrt{2 \pi s^{3}}} \exp \left(-\frac{(-x-\mu s)^{2}}{2 s}\right) d s
$$

and we easily deduce that:

$$
\mathbb{P}_{x}\left(T_{0}^{(B, \mu)}>\kappa\right) \underset{\kappa \rightarrow \infty}{\sim} \frac{x}{\sqrt{2 \pi \kappa^{3}}} e^{-\mu x-\kappa \mu^{2} / 2} .
$$

Then, as in the proof of Proposition 2.4:

$$
\begin{aligned}
\frac{\mathbb{E}_{x}\left[\mathbb{1}_{A_{t}} \mathbb{1}_{\left\{T_{0}^{(B, \mu)}>\kappa\right\}}\right]}{\mathbb{E}_{x}\left[\mathbb{1}_{\left\{T_{0}^{(B, \mu)}>\kappa\right\}}\right]} \underset{\kappa \rightarrow \infty}{\sim} \frac{\mathbb{E}_{x}\left[\mathbb{1}_{A_{t}} \mathbb{1}_{\left\{T_{0}^{(B, \mu)}>\kappa\right\}} \mathbb{E}_{\bar{B}(t)}\left(\mathbb{1}_{\left\{\widetilde{T}_{0}^{(B, \mu)}>\kappa-t\right\}}\right)\right]}{\mathbb{E}_{x}\left[\mathbb{1}_{\left\{T_{0}^{(B, \mu)}>\kappa\right\}}\right]} \\
\underset{\kappa \rightarrow \infty}{\sim} \frac{\mathbb{E}_{x}\left[\mathbb{1}_{\left.A_{t} \mathbb{1}_{\left\{T_{0}^{(B, \mu)}>\kappa\right\}}\left(\bar{B}(t) / \sqrt{2 \pi(\kappa-t)^{3}}\right) \exp \left(-\mu \bar{B}(t)-(\kappa-t) \mu^{2} / 2\right)\right]}^{\left(x / \sqrt{2 \pi \kappa^{3}}\right) \exp \left(-\mu x-\kappa \mu^{2} / 2\right)}\right.}{}
\end{aligned}
$$

and we deduce the expression of $M^{(B, \mu)}$. We prove the fact that it is a $\mathcal{F}^{(B, \mu)}$-martingale by using the classical Itô's formula. We write

$$
M^{(B, \mu)}(t)=\frac{e^{\mu x}}{x} \bar{B}(t) Y(t), \quad \text { where } Y(t)=e^{-\mu B(t)-\mu^{2} t / 2} .
$$

Clearly,

$$
Y(t)=e^{-\mu x}-\mu \int_{0}^{t} Y(s) d B(s)
$$

and then, by the integration by parts formula,

$$
M^{(B, \mu)}(t)=\frac{e^{\mu x}}{x} \bar{B}(t) Y(t)=1-\frac{e^{\mu x}}{x} \int_{0}^{t} Y(s)(1-\mu \bar{B}(s)) d B(s) .
$$

Let us now study the process $\{\bar{B}(t)\}_{t \geq 0}$ under $Q_{x}^{(B, \mu)}$. We consider an arbitrary function $f \in \mathrm{C}^{2}(\mathbb{R} ; \mathbb{R})$. Then, using Itô's formula and (3.12) we can write

$$
\begin{aligned}
& f(\bar{B}(t)) M^{(B, \mu)}(t)=f(x)+\int_{0}^{t}\left\{f(\bar{B}(s)) d M^{(B, \mu)}(s)+M^{(B, \mu)}(s) d f(\bar{B}(s))\right\} \\
+ & \left\langle f(\bar{B}), M^{(B, \mu)}\right\rangle(t)=f(x)+\int_{0}^{t}\left\{\frac{e^{\mu x}}{x} f(\bar{B}(s)) Y(s)(1-\mu \bar{B}(s))+f^{\prime}(\bar{B}(s)) M^{(B, \mu)}(s)\right\} d B(s) \\
& +\int_{0}^{t}\left\{\mu f^{\prime}(\bar{B}(s)) M^{(B, \mu)}(s)+\frac{1}{2} f^{\prime \prime}(\bar{B}(s)) M^{(B, \mu)}(s)+\frac{e^{\mu x}}{x} Y(s)(1-\mu \bar{B}(s)) f^{\prime}(\bar{B}(s))\right\} d s .
\end{aligned}
$$


Taking the expectation and using (3.11) we get

$$
\begin{aligned}
\mathbb{E}_{x}^{(B, \mu)}[f(\bar{B}(t))]=\mathbb{E}_{x}\left[f(\bar{B}(t)) M^{(B, \mu)}(t)\right] \\
=f(x)+\mathbb{E}_{x}\left[\int_{0}^{t}\left\{\frac{1}{2} f^{\prime \prime}(\bar{B}(s)) M^{(B, \mu)}(s)+\frac{e^{\mu x}}{x} Y(s) f^{\prime}(\bar{B}(s))\right\} d s\right] \\
=f(x)+\int_{0}^{t} \mathbb{E}_{x}\left[\mathcal{L} f(\bar{B}(s)) M^{(B, \mu)}(s)\right] d s=\mathbb{E}_{x}^{(B, \mu)}\left[f(x)+\int_{0}^{t} \mathcal{L} f(\bar{B}(s)) d s\right],
\end{aligned}
$$

where $\mathcal{L}=1 / 2 d^{2} / d x^{2}+1 / x d / d x$. We deduce that under $Q_{x}^{(B, \mu)}$, the infinitesimal generator of $\{\bar{B}(t)\}_{t \geq 0}$ is the infinitesimal generator of a Bessel process of dimension 3 .

\section{Bessel chains and other examples}

\subsection{Bessel chains}

We shall illustrate our results with two examples. In the discrete situation we consider the so-called Bessel random walk with index $\alpha>0$, while in the continuous parameter setting we adapt this example.

According to Theorem 2.1 one can obtain:

Corollary 4.1 Let $\left\{X_{n}\right\}_{n \geq 0}$ the Bessel random walk with index $\alpha>0$.

1. For any integer $n \geq 0$ and any event $A_{n} \in \mathcal{F}_{n},(2.7)$ is verified with the positive no uniformly integrable martingale

$$
M_{n}:=\frac{\Gamma(2 \alpha+1) X_{n} !}{\Gamma\left(X_{n}+2 \alpha+1\right)(2 \alpha+1)^{-U_{n}+\mathbb{1}_{\left\{X_{n}=0\right\}}} .}
$$

2. Let $Q_{0}$ be the probability measure on $\left(\Omega, \mathcal{F}_{\infty}\right)$ given by $(2.9)$. Then, under $Q_{0},\left\{X_{n}\right\}_{n \geq 0}$ is a recurrent birth and death chain with transition probabilities given by:

$$
\tilde{p}_{0}=1 \quad \text { and } \quad \tilde{p}_{\ell}:=\frac{\ell+1}{2 \ell+2 \alpha+1}, \tilde{q}_{\ell}:=\frac{\ell+2 \alpha}{2 \ell+2 \alpha+1}, \text { for } \ell \geq 1 .
$$

Proof. We compute $R_{n}, \forall n \geq 0$. First, by (2.3):

$$
\gamma_{\ell}=\prod_{i=1}^{\ell} \frac{q_{i}}{p_{i}}=\prod_{i=1}^{\ell} \frac{i}{i+2 \alpha+1}=\Gamma(2 \alpha+2) \frac{\Gamma(\ell+1)}{\Gamma(\ell+2 \alpha+2)}=(2 \alpha+1) \beta(2 \alpha+1, \ell+1) .
$$

Then, by (3.2), for $n \geq 1$ :

$R_{n}=(2 \alpha+1) \sum_{\ell \geq n} \beta(2 \alpha+1, \ell+1)=(2 \alpha+1) \sum_{\ell \geq 0} \beta(2 \alpha+1, \ell+n+1)=(2 \alpha+1) \beta(2 \alpha, n+1)$,

where we used the following equality $\sum_{k \geq 0} \beta(x, y+k)=\beta(x-1, y)$ (see for instance, [3], p. 950). Hence,

$$
R_{n}=\frac{(2 \alpha+1) \Gamma(2 \alpha) n !}{\Gamma(n+2 \alpha+1)}, \text { for } n \geq 1, \quad \text { and } \quad R_{0}=S=1+R_{1}=\frac{2 \alpha+1}{2 \alpha} .
$$


The expression (4.1) is then a consequence of (4.3) and (2.8). Furthermore, according to (2.10), $\tilde{p}_{k}=p_{k} R_{k+1} / R_{k}$, and using again (4.3) we obtain (4.2).

\section{Remark 4.1}

In [11] one considers the penalization of the transient continuous Bessel process $\left\{\mathrm{R}_{t}\right\}_{t \geq 0}$ with dimension $\mathrm{d}>2$ (or of index $\mathrm{n}=\mathrm{d} / 2-1>0$ ) by its sojourn time in an interval. Set, for $r>0, \mathrm{U}_{t}^{(r)}=\int_{0}^{t} \mathbb{1}_{[0, r]}\left(\mathrm{R}_{s}\right) d s(0 \leq t \leq \infty)$. One studies the limit

$$
\lim _{t \rightarrow \infty} \frac{\mathbb{E}_{x}\left[\mathbb{1}_{A_{s}} \mathbb{1}_{\left\{\mathrm{U}_{\infty}^{(r)}>t\right\}}\right]}{\mathbb{E}_{x}\left[\mathbb{1}_{\left\{\mathrm{U}_{\infty}^{(r)}>t\right\}}\right]}=\mathbb{E}_{x}\left[\mathbb{1}_{\left.A_{s} \mathrm{M}_{s}^{(r)}\right]}\right]=: \mathbf{Q}_{x}^{(r)}\left(A_{s}\right), A_{s} \in \mathcal{F}_{s} \quad(s \text { fixed }) .
$$

One proves in [11] that:

- the positive martingale $\mathrm{M}_{s}^{(r)}$ is given by $\mathrm{M}_{t}^{(r)}:=h^{(r)}\left(\mathrm{R}_{t}\right) \exp \left(z_{1} \mathrm{U}_{t}^{(r)} / 2 r^{2}\right)$, where $h^{(r)}(z):=\Gamma(\mathrm{n}+1)\left[\left(2 r / z_{1} z\right)^{\mathrm{n}} \mathrm{J}_{\mathrm{n}}\left(z_{1} z / r\right) \mathbb{1}_{0 \leq z \leq r}+\left(2 r^{2} / z_{1} z^{2}\right)^{\mathrm{n}} \mathrm{J}_{\mathrm{n}}\left(z_{1}\right) \mathbb{1}_{z \geq r}\right]$, and $z_{1}$ is the first positive zero of the Bessel function $J_{\mathrm{n}-1}$.

- for $x \geq r$, under $\mathrm{Q}_{x}^{(r)}$, the process $\left\{\mathrm{R}_{t}\right\}_{t \geq 0}$ has the infinitesimal generator $\mathrm{L}:=1 / 2 d^{2} / d x^{2}+$ $(1-2 \mathrm{n}) / x d / d x$. This process is recurrent and behaves, in a certain sense, as $r \rightarrow 0$, as a Bessel process of dimension $4-\mathrm{d}$ (or of index $-\mathrm{n}$ ).

We return to the case of Bessel random walk with index $\alpha>-1$ and transition probabilities given by (2.12) and we illustrate now a similar phenomenon as for the continuous Bessel process. Let us remak that the Bessel random walk could also be characterized by the following transition probabilities:

$$
\begin{aligned}
\mathbb{P}\left(X_{n+1}=\ell-1 \mid X_{n}=\ell\right) & =\frac{1}{2}\left(1-\frac{\alpha+1 / 2}{\ell+\alpha+1 / 2}\right) \\
& \mathbb{P}\left(X_{n+1}=\ell+1 \mid X_{n}=\ell\right)=\frac{1}{2}\left(1+\frac{\alpha+1 / 2}{\ell+\alpha+1 / 2}\right), \text { for } \ell, n \geq 1 \text { integers. }
\end{aligned}
$$

Clearly, if $\alpha \in(0,1) \backslash\{1 / 2\}$ (or of dimension $\delta \in(2,4) \backslash\{3\}$ ),

$$
Q\left(X_{n+1}=\ell-1 \mid X_{n}=\ell\right)=\frac{1}{2}\left(1-\frac{-\alpha+\frac{1}{2}}{\ell-\alpha+\frac{1}{2}}\right)+\frac{H(\ell)}{2},
$$

with

$$
H(\ell)=\frac{\alpha / 2-\alpha^{2}}{(\ell-\alpha+1 / 2)(\ell+\alpha+1 / 2)}
$$

As $\ell \rightarrow \infty$, we can write

$$
Q\left(X_{n+1}=\ell-1 \mid X_{n}=\ell\right)=\frac{1}{2}\left(1-\frac{-\alpha+\frac{1}{2}}{\ell-\alpha+\frac{1}{2}}\right)+o(1 / \ell) .
$$

In other words, if $\alpha \neq 1 / 2$, the behaviour of the chain $\left\{X_{n}\right\}_{n \geq 0}$ under $Q$, is that of a Bessel random walk with index $\alpha^{\prime}=-\alpha$ (or with dimension $\delta^{\prime}=4-\delta \in(0,2) \backslash\{1\}$ ), at least for large states $\ell$. If $\alpha=1 / 2$, the chain $\left\{X_{n}\right\}_{n \geq 0}$ under $Q$ is a standard symmetric random walk. 
Let us turn now to the continuous parameter example which is built using the previous example. Let us call it Bessel jump process with index $\alpha>0$. It is a pure jump process $\{X(t)\}_{t \geq 0}$ with birth and death rates:

$$
b_{\ell}=\ell+2 \alpha+1, \quad d_{\ell}=\ell, \text { for } \ell \geq 0 \text { integer. }
$$

We can state a consequence of Theorem 2.2:

\section{Corollary 4.2}

1. For any $t \geq 0$ and any event $A_{t} \in \mathcal{F}_{t},(2.19)$ is verified with the positive no uniformly integrable martingale

$$
M(t):=\frac{2 \alpha}{2 \alpha+1} e^{2 \alpha V(t)} \frac{\Gamma(2 \alpha+1) X(t) !}{\Gamma(2 \alpha+X(t)+1)} .
$$

2. Let $Q$ be the probability measure on $\left(\Omega, \mathcal{F}_{\infty}\right)$ given by $(2.21)$. Then, under $Q,\left\{X_{t}\right\}_{t \geq 0}$ is a recurrent birth and death chain with transition probabilities given by:

$$
\tilde{b}_{\ell}:=\ell+1, \ell \geq 0, \quad \tilde{d}_{\ell}:=\ell+2 \alpha, \ell \geq 1 .
$$

The proof of this corollary is based on the following fact : the underlying jump chain associated to $\{X(t)\}_{t \geq 0}$ is a Bessel random walk with index $\alpha$. The remaining details are left to the reader.

\section{$4.2 \quad$ Other examples}

Let us state other natural questions: assuming the hypothesis of Theorem 2.2, the process $\{X(t)\}_{t \geq 0}$, under $Q$, is it recurrent positive? Does there exist an invariant probability distribution? The answer at these questions is no. Here are two simple examples.

Set, for $\ell \geq 0, b_{\ell}:=a^{\ell}, d_{\ell}:=a^{2 \ell}$, where $0<a<1$. Therefore, the associated birth and death process is transient since

$$
\gamma_{n}=\prod_{\ell=1}^{n} a^{\ell}=a^{n(n+1) / 2}, \quad S=\sum_{n \geq 0} a^{n(n+1) / 2}<\infty .
$$

Again, the process is recurrent under $Q$, by Theorem 2.2. Using (2.22) we get

$$
\tilde{b}_{\ell}=b_{\ell} \frac{R_{\ell+1}}{R_{\ell}}=a^{\ell} \frac{R_{\ell+1}}{R_{\ell}}, \quad \tilde{d}_{\ell}=d_{\ell} \frac{R_{\ell-1}}{R_{\ell}}=a^{2 \ell} \frac{R_{\ell-1}}{R_{\ell}} .
$$

We compute

$$
\sum_{j \geq 1} \prod_{\ell=1}^{j} \frac{\tilde{b}_{\ell-1}}{\tilde{d}_{\ell}}=\sum_{j \geq 1}\left(\frac{R_{j}}{S}\right)^{2} \prod_{\ell=1}^{j} \frac{1}{a^{\ell+1}}=\frac{1}{S^{2}} \sum_{j \geq 1} \frac{R_{j}^{2}}{a^{\left(j^{2}+3 j\right) / 2}}=\frac{1}{S^{2}} \sum_{j \geq 1} U_{j},
$$


where

$$
U_{j}:=\frac{1}{a^{\left(j^{2}+3 j\right) / 2}}\left(\sum_{\ell \geq j} a^{\ell(\ell+1) / 2}\right)^{2} .
$$

Since

$$
\frac{U_{j+1}}{U_{j}}=a^{(2 j-4) / 2} \frac{a^{(j+1)(j+2)}}{a^{j(j+1)}}\left(\frac{\sum_{\ell \geq 0} a^{\ell(\ell+2 j+3) / 2}}{\sum_{\ell \geq 0} a^{\ell(\ell+2 j+1) / 2}}\right)^{2}=a^{3 j}\left(\frac{\sum_{\ell \geq 0} a^{\ell(\ell+2 j+3) / 2}}{\sum_{\ell \geq 0} a^{\ell(\ell+2 j+1) / 2}}\right)^{2}
$$

and, by dominated convergence theorem,

$$
\lim _{j \rightarrow \infty} \frac{\sum_{\ell \geq 0} a^{\ell(\ell+2 j+3) / 2}}{\sum_{\ell \geq 0} a^{\ell(\ell+2 j+1) / 2}}=1,
$$

then $\lim _{j \rightarrow \infty} U_{j+1} / U_{j}=0$. By D'Alembert criterion, the series $\left(1 / S^{2}\right) \sum_{j \geq 1} U_{j}$ converges and therefore the necessary condition for the existence of an invariant distribution (see, for instance [6], p. 78 or [10], p. 412) is satisfied. Hence, in this case there exists an invariant distribution for the process $\{X(t)\}_{t \geq 0}$ under $Q$.

Set, for $\ell \geq 0, b_{\ell}:=a^{\ell}, d_{\ell}:=a^{\ell+1}$, where $0<a<1$. Again, the associated birth and death process is transient since

$$
\gamma_{n}=\prod_{\ell=1}^{n} a=a^{n}, \quad S=\sum_{n \geq 0} \gamma_{n}=\frac{1}{1-a}<\infty .
$$

Using (2.22) we obtain

$$
\tilde{b}_{\ell}=b_{\ell} \frac{R_{\ell+1}}{R_{\ell}}=b_{\ell} \frac{a^{\ell+1}}{a^{\ell}}=a^{\ell+1}, \quad \tilde{d}_{\ell}=d_{\ell} \frac{R_{\ell-1}}{R_{\ell}}=d_{\ell} \frac{a^{\ell-1}}{a^{\ell}}=a^{\ell} .
$$

On the one hand, by Theorem 2.2 one knows that the process is recurrent under $Q$. On the other hand:

$$
\sum_{j \geq 1} \prod_{\ell=1}^{j} \frac{\tilde{b}_{\ell-1}}{\tilde{d}_{\ell}}=\sum_{j \geq 1} 1=\infty
$$

Hence in this case there is not invariant distribution for $\{X(t)\}_{t \geq 0}$ under $Q$.

Let us note that in previous examples there is no explosion. A natural question is then the following : if the process explodes is it possible to prove the existence of an invariant distribution? Again the answer is negative as we can see below.

Let us recall, according to [9] p. 90 , that $\{X(t)\}_{t \geq 0}$ can explode if $\sup _{\ell \geq 0} b_{\ell}+d_{\ell}=\infty$. Set, for $\ell \geq 0, b_{\ell}=(1+\ell)^{a}$ and $d_{\ell}=\ell^{a}$, with $a>1$. Then, obviously $\sup _{\ell \geq 0} b_{\ell}+d_{\ell}=\infty$, and

$$
\gamma_{n}=\prod_{\ell=1}^{n} \frac{\ell^{a}}{(1+\ell)^{a}}, \quad S=\sum_{\ell \geq 0} \frac{1}{(1+\ell)^{a}}<\infty .
$$

As previously, we have:

$$
\sum_{j \geq 1} \prod_{\ell=1}^{j} \frac{\tilde{b}_{\ell-1}}{\tilde{d}_{\ell}}=\sum_{j \geq 1}\left(\frac{R_{j}}{S}\right)^{2} \prod_{\ell=1}^{j} \frac{b_{\ell-1}}{d_{\ell}}=\frac{1}{S^{2}} \sum_{j \geq 1}\left(\sum_{\ell \geq j} \frac{1}{(1+\ell)^{a}}\right)^{2} .
$$


Since

$$
\sum_{\ell \geq j} \frac{1}{(1+\ell)^{a}} \underset{j \rightarrow \infty}{\sim} \int_{j}^{\infty} \frac{1}{(1+y)^{a}}=\frac{1}{(a-1)(j+1)^{a-1}}
$$

the series $\sum_{j \geq 1} \prod_{\ell=1}^{j} \tilde{b}_{\ell-1} / \tilde{d}_{\ell}$ behaves as the series $\sum_{j \geq 1}(j+1)^{2-2 a}$, that is convergent if $a>3 / 2$ and divergent if $a \leq 3 / 2$.

Finally, the explosion is not a criterion for the existence of an invariant distribution.

Acknowledgement: We thank Bernard Roynette for many valuable discussions on the subject and especially about Proposition 2.5 and Remark 4.1.

\section{References}

[1] Debs, P. (2007). Pénalisation de la marche aléatoire standard par une fonction du maximum unilatère, du temps local en zéro et de la longueur des excursions. Preprint IECN.

[2] Feller, W. (1970). An introduction to probability theory and its applications vol. 1, 3rd edition, Wiley, New York.

[3] Gradstein, I.S., Ryzhik, I.M. (1980). Table of integrals, Series and Products, Academic Press New York.

[4] Karatzas, I., Shreve, S.E. (1991). Brownian motion and stochastic calculus, 2nd edition, Springer New York.

[5] Klebaner, F.C. (2005). Introduction to Stochastic Calculus with Applications 2nd edition, Imperial College Press, London.

[6] Lawler, G. (2006). Introduction to Stochastic Processes 2nd edition, Chapman \& Hall/CRC, Boca Raton.

[7] Le Gall, J.-F. (1986). Une approche élémentaire des théorémes de décomposition de Williams. Séminaire de Probabilités, XX, 1984/85, 447-464, Lecture Notes in Math. 1204, Springer, Berlin.

[8] Mishchenko, A.S. (2006). A discrete Bessel process and its properties. Theory Probab. Appl. 50, 700-709.

[9] Norris, J. (1998). Markov chains. 2nd printing, Cambridge University Press, Cambridge.

[10] Resnick, S.I. (2005). Adventures in Stochastic Processes, 4th printing, Birkhäuser, Boston, 2005.

[11] Roynette, B., Yor, M. (2007). Brownian penalizations: metatheorem and correct results. Book in progress.

[12] Roynette, B., Vallois, P., Yor, M. (2006). Pénalisations et quelques extensions du théorème de Pitman, relatives au mouvement brownien et à son maximum unilatère, Séminaire de Probabilités XXXVIII : In memoriam Paul-André Meyer, 305-336, Lecture Notes in Math. 1874, Springer, Berlin. 